\title{
Generalized Stirling Numbers and Hyper-Sums of Powers of Binomial Coefficients
}

\author{
Claudio de J. Pita Ruiz V. \\ Department of Mathematics \\ Universidad Panamericana \\ Mexico City, Mexico \\ cpita@up.edu.mx
}

Submitted: Oct 9, 2013; Accepted: Dec 16, 2013; Published: Jan 12, 2014

Mathematics Subject Classifications: 05A10, 11B73

\begin{abstract}
We work with a generalization of Stirling numbers of the second kind related to the boson normal ordering problem (P. Blasiak et al.). We show that these numbers appear as part of the coefficients of expressions in which certain sequences of products of binomials, together with their partial sums, are written as linear combinations of some other binomials. We show that the number arrays formed by these coefficients can be seen as natural generalizations of Pascal and Lucas triangles, since many of the known properties on rows, columns, falling diagonals and rising diagonals in Pascal and Lucas triangles, are also valid (some natural generalizations of them) in the arrays considered in this work. We also show that certain closed formulas for hyper-sums of powers of binomial coefficients appear in a natural way in these arrays.
\end{abstract}

Keywords: generalized Stirling numbers, powers of binomial coefficients

\section{Introduction}

Along the years, Stirling numbers have demonstrated to be a fundamental tool ('of the greatest utility' [17]) for dealing with combinatorial problems. We can find now many research works pursuing generalizations (in some sense) of Stirling numbers of both kinds (some of them accompanied with the corresponding combinatorial interpretations). We mention some: L. Carlitz [6] studied a type of $\lambda$-weighted Stirling numbers/polynomials which have demonstrated to be useful to understand different mathematical problems (see for example [12] and [18]); W. Lang [21] considered a (one-parameter) generalization 
of Stirling numbers, by using the language of 'infinitesimal transformations' (with some flavor from Lie groups theory). Lang himself [20] studied the corresponding combinatorial interpretation of his generalized Stirling numbers. One more generalized version of Stirling numbers appeared in the work of T. Mansour et al. [22], and one yet more in the work of M. Dziemiańczuk [11]. We mention also the work of Tian-Xiao He [16], which presents a generalization that includes some other known generalizations of Stirling numbers. (See also Remark 1 at the end of this section.) Among all these works, there is one concerning Stirling numbers of the second kind, which has a natural connection with certain products of binomial coefficients. Having this connection as starting point we construct some number arrays which are natural generalizations of Pascal and Lucas triangles (and also of the corresponding results of the previous work [24]). To study these number arrays is the main theme of this article. The mentioned generalization of Stirling numbers of the second kind appears in the works of P. Blasiak et al. [2, 4, 23] (see also [1]). (Two works relating this generalization with other combinatorial-physics objects are [26, 27]; two works about the combinatorial interpretations of these generalized Stirling numbers are $[3,10]$.) We devote the rest of this section to present the basic facts of Blasiak's generalized Stirling numbers of the second kind.

Throughout the work, $p$ denotes a given natural number. To keep the original author's notation, we use $r$ and $s$ to denote positive integers such that $r \geqslant s$. The $(r, s)$-Stirling numbers of the second kind $S_{r, s}(p, k)$ (or simply generalized Stirling numbers of the second kind), are introduced in the P. Blasiak's main work [2] as certain coefficients appearing in the so-called boson normal ordering problem. Roughly speaking, the numbers $S_{r, s}(p, k)$ are determined by the following equation:

$$
\left(x^{r} \frac{d^{s}}{d x^{s}}\right)^{p}=x^{p(r-s)} \sum_{j=s}^{p s} S_{r, s}(p, j) x^{j} \frac{d^{j}}{d x^{j}} .
$$

For example, if we set $r=4, s=3$ and $p=2$, formula (1) looks as

$$
\left(x^{4} \frac{d^{3}}{d x^{3}}\right)^{2}=x^{2} \sum_{j=3}^{6} S_{4,3}(2, j) x^{j} \frac{d^{j}}{d x^{j}} .
$$

Letting act both sides of $(2)$ on a $C^{6}(\mathbb{R})$ function $y=y(x)$, the left-hand side becomes

$$
x^{4} \frac{d^{3}}{d x^{3}}\left(x^{4} \frac{d^{3} y}{d x^{3}}\right)=x^{2}\left(24 x^{3} \frac{d^{3} y}{d x^{3}}+36 x^{4} \frac{d^{4} y}{d x^{4}}+12 x^{5} \frac{d^{5} y}{d x^{5}}+x^{6} \frac{d^{6} y}{d x^{6}}\right)
$$

so we have that $S_{4,3}(2,3)=24, S_{4,3}(2,4)=36, S_{4,3}(2,5)=12$ and $S_{4,3}(2,6)=1$.

By convention one takes $S_{r, s}(p, 0)=\delta_{p, 0}$. It can be shown that $S_{r, s}(p, j) \neq 0$ if and only if $s \leqslant j \leqslant s p$, and that $S_{r, s}(p, p s)=1$ for $p \in \mathbb{N}$. The standard Stirling numbers of the second kind $S(p, j)$ correspond to the case $r=s=1$. An explicit formula for $S_{r, s}(p, j)$ is

$$
S_{r, s}(p, j)=\frac{(-1)^{j}}{j !} \sum_{i=s}^{j}(-1)^{i}\left(\begin{array}{l}
j \\
i
\end{array}\right) \prod_{l=1}^{p}(i+(l-1)(r-s))^{\underline{s}}
$$


where $m^{\underline{s}}=\prod_{l=0}^{s-1}(m-l)$ is the falling factorial. From (3) one can see at once that $S_{s, s}(p, s)=(s !)^{p-1}$. (Note that when $r=s=1$, formula (3) is the known explicit formula for Stirling numbers of the second kind $S(p, k)=\frac{(-1)^{k}}{k !} \sum_{i=1}^{k}(-1)^{i}\left(\begin{array}{c}k \\ i\end{array}\right) i^{p}$.) It is possible to see from (1) that

$$
\prod_{j=1}^{p}(x+(j-1)(r-s))^{\underline{s}}=\sum_{k=s}^{p s} S_{r, s}(p, k) x^{\underline{k}} .
$$

Observe that the case $s=r$ of (4) is

$$
\left(x^{\underline{r}}\right)^{p}=\sum_{k=r}^{p r} S_{r, r}(p, k) x^{\underline{k}},
$$

and that the case $r=1$ of (5) is the well-known expression

$$
x^{p}=\sum_{k=1}^{p} S(p, k) x^{\underline{k}},
$$

in which the Stirling numbers of the second kind $S(p, k)$ appear as the connecting coefficients when $x^{p}$ is written as a linear combination of the falling factorials $x^{\frac{k}{k}}, k=1,2, \ldots, p$.

Formula (4.56) -corrected- from Blasiak [2], gives us the recurrence relation

$$
S_{r, s}(p+1, k)=\sum_{i=0}^{s}\left(\begin{array}{c}
p(r-s)+k+i-s \\
i
\end{array}\right) s^{\underline{i}} S_{r, s}(p, k+i-s) .
$$

(When $r=s=1$ we have the known recurrence $S(p+1, k)=S(p, k-1)+k S(p, k)$ for the usual Stirling numbers of the second kind.)

An interesting particular case is when $r=2 s$. We claim that

$$
S_{2 s, s}(p, k)=\frac{(s p) !}{k !}\left(\begin{array}{c}
s(p-1) \\
k-s
\end{array}\right) .
$$

Clearly this formula is true for $p=1$. Assuming the formula is true for a given $p \in \mathbb{N}$, we have according to $(7)$ that

$$
S_{2 s, s}(p+1, k)=\sum_{i=0}^{s}\left(\begin{array}{c}
p s+k+i-s \\
i
\end{array}\right) s^{\underline{i}} S_{2 s, s}(p, k+i-s) .
$$

By using the induction hypothesis together with some algebraic manipulations, we can write (9) as

$$
S_{2 s, s}(p+1, k)=\frac{(s(p+1)) !}{k !} \frac{1}{\left(\begin{array}{c}
s(p-1)+k \\
k
\end{array}\right)} \sum_{i=0}^{s}\left(\begin{array}{c}
s \\
i
\end{array}\right)\left(\begin{array}{c}
s p \\
k-i
\end{array}\right)\left(\begin{array}{c}
s p+k-i \\
k-i-s
\end{array}\right) .
$$

But identity (6.47) from Gould's book [13] gives us that

$$
\sum_{i=0}^{s}\left(\begin{array}{l}
s \\
i
\end{array}\right)\left(\begin{array}{c}
s p \\
k-i
\end{array}\right)\left(\begin{array}{c}
s p+k-i \\
k-i-s
\end{array}\right)=\left(\begin{array}{c}
s p \\
k-s
\end{array}\right)\left(\begin{array}{c}
s(p-1)+k \\
k
\end{array}\right),
$$


so we conclude finally from (10) and (11) that

$$
S_{2 s, s}(p+1, k)=\frac{(s(p+1)) !}{k !}\left(\begin{array}{c}
s p \\
k-s
\end{array}\right),
$$

proving our claim (8). (The case $s=1$ of $(8)$ is $\frac{p !}{k !}\left(\begin{array}{c}p-1 \\ k-1\end{array}\right)$, corresponding to the so-called (unsigned) Lah numbers [25, A008297].)

In [2] there are tables for $S_{r, s}(p, k)$ with $r=1,2,3,1 \leqslant s \leqslant r$ and some small values of $p$ and the corresponding $s \leqslant k \leqslant p s$. Some $S_{4, s}(p, k)$ for $1 \leqslant s \leqslant 4$ are the following:

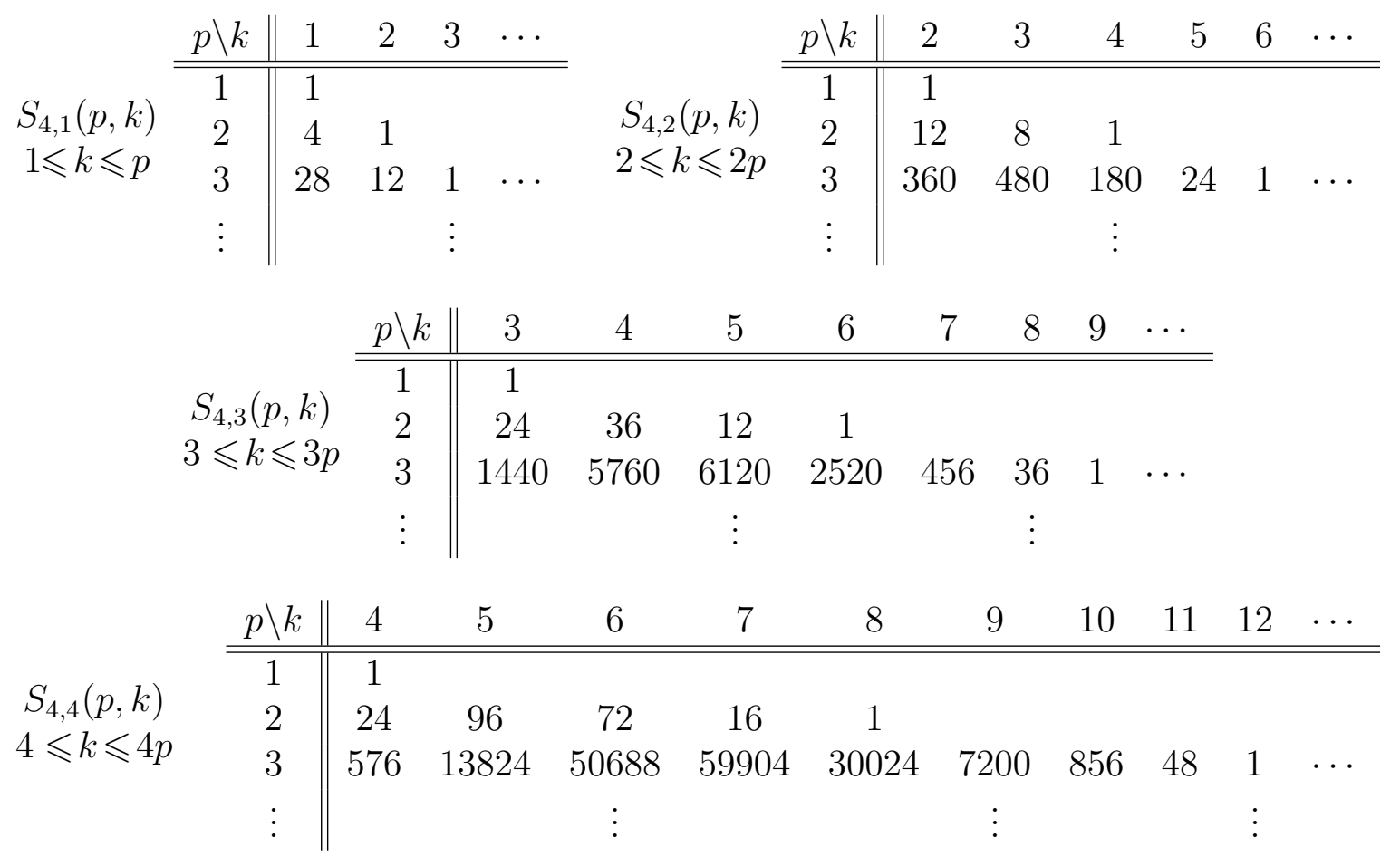

Recall that the convolution of the sequences $a_{n}$ and $b_{n}$ is defined as the sequence $a_{n} * b_{n}=\sum_{t=0}^{n} a_{t} b_{n-t}$; in particular $a_{n} * 1$ is the sequence of partial sums of $a_{n}$, and for a non-negative integer $l$, the sequence $a_{n} *^{l} 1$ is defined recursively as $a_{n} *^{0} 1=a_{n}$ and $a_{n} *^{l} 1=\left(a_{n} *^{l-1} 1\right) * 1$.

Beginning with the sequence $n^{p}$ (where $p \in \mathbb{N}$ is given), written as

$$
n^{p}=\sum_{j=1}^{p} j ! S(p, j)\left(\begin{array}{l}
n \\
j
\end{array}\right)
$$

(see formula (6.10) in [15]) and the partial sums of it, namely $n^{p} *^{l} 1$, we studied in [24] the number arrays formed by the coefficients resulting when we write these sequences as linear combinations of binomial coefficients. We called them $p$-arrays. It turns out that these arrays are natural generalizations of Pascal's triangle (case $p=1$ ) and Lucas triangle (case $p=2$ ). We showed that many of the known properties of Pascal and Lucas triangles 
(the expected generalizations of those properties) are valid for the $p$-arrays, including the property on rising diagonals (recall that the sums of rising diagonals of Pascal's triangle are Fibonacci numbers, and in the Lucas triangle are Lucas numbers). We showed that the sums of the rising diagonals in a $p$-array is equal to some constant (the Stirling-Bernoulli transform of Fibonacci numbers, that depends only on $p$; see [25, A050946]) times a Fibonacci number, when $p$ is odd, and times a Lucas number, when $p$ is even. In this work we generalize the above results. Beginning with the sequence $\prod_{j=1}^{p}\left(\begin{array}{c}n+(j-1)(r-s) \\ s\end{array}\right)$, where $r, s \in \mathbb{N}$ are given, and $1 \leqslant s \leqslant r$, we show that a similar formula to (13) is valid, namely

$$
\prod_{j=1}^{p}\left(\begin{array}{c}
n+(j-1)(r-s) \\
s
\end{array}\right)=\frac{1}{(s !)^{p}} \sum_{j=s}^{p s} j ! S_{r, s}(p, j)\left(\begin{array}{l}
n \\
j
\end{array}\right) .
$$

Observe that in the case $r=s=1$, formula (14) is (13), and in the case $r=s$ formula (14) looks as

$$
\left(\begin{array}{l}
n \\
s
\end{array}\right)^{p}=\frac{1}{(s !)^{p}} \sum_{j=s}^{p s} j ! S_{s, s}(p, j)\left(\begin{array}{l}
n \\
j
\end{array}\right),
$$

which gives us the $p$-th power of the binomial coefficient $\left(\begin{array}{l}n \\ s\end{array}\right)$ written as a linear combination of the binomials $\left(\begin{array}{l}n \\ j\end{array}\right), s \leqslant j \leqslant s p$.

Moreover, we consider the partial sums of the sequence $\prod_{j=1}^{p}\left(\begin{array}{c}n+(j-1)(r-s) \\ s\end{array}\right)$ and show that they can be written as linear combination of binomial coefficients according to

$$
\begin{aligned}
& \left(\prod_{j=1}^{p}\left(\begin{array}{c}
n+(j-1)(r-s) \\
s
\end{array}\right)\right) *^{k-p r+s-1} 1 \\
= & \frac{1}{(s !)^{p}} \sum_{j=s}^{k-p(r-s)+s-1} \sum_{i=s}^{j}\left(\begin{array}{c}
k-p r+s-1 \\
j-i
\end{array}\right) i ! S_{r, s}(p, i)\left(\begin{array}{l}
n \\
j
\end{array}\right),
\end{aligned}
$$

where $k \geqslant p r-s+1$. With the coefficients

$$
a_{k, j}^{(r, s)}=\frac{1}{(s !)^{p}} \sum_{i=s}^{j}\left(\begin{array}{c}
k-p r+s-1 \\
j-i
\end{array}\right) i ! S_{r, s}(p, i)
$$

we form the $(r, s, p)$-arrays (with $k$ for lines and $j$ for columns), which generalize the $p$-arrays of [24], and then also generalize the Pascal's triangle (case $r=s=p=1$ ) and Lucas triangle (case $r=s=1, p=2$ ). Observe that the case $r=s$ of (16) give us the 'hyper-sum' (partial sums) of powers of binomial coefficients expressed as a linear combination of binomial coefficients, namely

$$
\left(\begin{array}{l}
n \\
s
\end{array}\right)^{p} *^{k-p r+s-1} 1=\frac{1}{(s !)^{p}} \sum_{j=s}^{k+s-1} \sum_{i=s}^{j}\left(\begin{array}{c}
k-p(s-1)-1 \\
j-i
\end{array}\right) i ! S_{s, s}(p, i)\left(\begin{array}{l}
n \\
j
\end{array}\right) .
$$


Expressions like (18) will appear several times in this work, with variants in the righthand side. What we have in the left-hand side of (18) is a (partial) sum of (partial) sums of the sequence of $p$-th powers of the binomial coefficients $\left(\begin{array}{l}n \\ s\end{array}\right)$, in which $s$ is fixed and $n$ is the running index. To find closed formulas for sums of powers of binomial coefficients of the type $\sum_{k=0}^{n}\left(\begin{array}{l}n \\ k\end{array}\right)^{p}$ are difficult problems (it is known that in some cases these formulas do not exist; see for example [5]). Our sums of powers of binomial coefficients do not belong to the difficult ones, but we believe that they are new results.

In section 2 we prove the main results of the work (formulas (14) and (16), in propositions 2 and 3, respectively). We also exhibit in this section the first few rows and columns of some $(r, s, p)$-arrays. In section 3 we mention some of the 'easy' properties of the $(r, s, p)$-arrays, related to their rows, columns, and falling diagonals. The proofs of most of these properties are left as easy exercises to the reader. In section 4 we consider the rising diagonals of the $(r, s, p)$-arrays. As in the previous work [24], this is the most important section of the article, and justifies that our $(r, s, p)$-arrays can be seen as generalizations of Pascal and Lucas triangles. In this section we consider only $(r, s, p)$-arrays in which $r=s$ (see Remark 9). We prove that in the sums of rising diagonals in a $(r, r, p)$ array appear Fibonacci numbers when $r$ is even or $r$ and $p$ are odd (this includes the case $r=1$ and $p$ odd of [24]), or Lucas numbers when $r$ is odd and $p$ is even (this includes the case $r=1$ and $p$ even of [24]), multiplied by a constant that depends only on $r$ and $p$. The corresponding sequences of these constants (with $p \in \mathbb{N}$ and $r$ is fixed) generalize the sequence of Stirling-Bernoulli transform of Fibonacci numbers mentioned before. Finally, in section 5 we use a result of section 4 to obtain a different version of (15). It turns out that this new version can be simplified to a different expression for $\left(\begin{array}{l}n \\ s\end{array}\right)^{p}$ similar to (15) but simpler than it.

Remark 1. We would like to do some comments on the direction presented in [8] for a generalization of Stirling numbers (see also the previous works related to this generalization $[9,19]$, and the later work [7] containing $q$-versions of it). Certainly what is presented in [8] is different to the direction contained in Blasiak's works, but we think it is worth to mention it since we can see some similar mathematical ingredients in both (besides the non-mathematical fact - but a coincidence, after all - that the generalization presented in [8] is also a two parameters one, and these parameters are also denoted by $r$ and $s$ - with nothing to do with Blasiak's parameters $r$ and $s-)$.

The classical definition of Stirling numbers of the second kind $S(n, k)$ in (6) can be generalized if the 'central' $x(=x+0)$ of the left-hand side, is replaced by the 'non-central' $x+r$, namely

$$
(x+r)^{n}=\sum_{k=0}^{n} S_{r}(n, k) x^{\underline{k}} .
$$

The numbers $S_{r}(n, k)$ are called non-central Stirling numbers of the second kind. So- 
me examples are the following

$$
\begin{array}{lll}
S_{r}(1,0)=r & S_{r}(2,0)=r^{2} & S_{r}(3,0)=r^{3} \\
S_{r}(1,1)=1 & S_{r}(2,1)=2 r+1 & S_{r}(3,1)=3 r^{2}+3 r+1 \\
& S_{r}(2,2)=1 & S_{r}(3,2)=3 r+3 \\
& & S_{r}(3,3)=1
\end{array}
$$

If now the powers $(x+r)^{n}$ of the left-hand side of (19) are replaced by the noncentral re-scaled falling factorials $(s x+r)^{\underline{n}}$, we obtain some new coefficients in the linear combination of the right-hand side of (19). We have

$$
(s x+r)^{\underline{n}}=\sum_{k=0}^{n} C_{r, s}(n, k) x^{\underline{k}} .
$$

The numbers $C_{r, s}(n, k)$ are called non-central generalized factorial coefficients (or Gould-Hopper numbers). Observe that (20) share with (4) the fact that both formulas express certain falling factorials (those of the corresponding left-hand sides) as linear combinations of some other falling factorials (those of the corresponding right-hand sides). So we can see the non-central generalized factorial coefficients $C_{r, s}(n, k)$ of (20) as the objects playing a similar role to the generalized Stirling numbers of the second kind $S_{r, s}(p, k)$ of (4). Some examples are the following

$$
\begin{aligned}
& C_{r, s}(1,0)=r \\
& C_{r, s}(1,1)=s \\
& C_{r, s}(2,0)=r(r-1) \\
& C_{r, s}(2,1)=s(2 r+s-1) \\
& C_{r, s}(2,2)=s^{2} \\
& C_{r, s}(3,0)=r(r-1)(r-2) \\
& C_{r, s}(3,1)=s\left(3 r^{2}+3 r(s-2)+(s-1)(s-2)\right) \\
& C_{r, s}(3,2)=3 s^{2}(r+s-1) \\
& C_{r, s}(3,3)=s^{3}
\end{aligned}
$$

We have the explicit formula [8, p. 335, ex. 35]

$$
C_{r, s}(n, k)=\frac{1}{k !} \sum_{j=0}^{k}(-1)^{k+j}\left(\begin{array}{l}
k \\
j
\end{array}\right)(s j+r)^{\underline{n}} .
$$

A connection of $C_{r, s}(n, k)$ with $S_{r}(n, k)$ is the following

$$
\lim _{s \rightarrow \infty} s^{-n} C_{r, s}(n, k)=S_{\rho}(n, k),
$$

where $\lim _{s \rightarrow \infty}\left(r s^{-1}\right)=\rho$ [8, p. 335, ex. 37]. The particular case $s=-1$ of (20) gives us the coefficients $C_{r,-1}(n, k)$, called non-central Lah numbers and denoted by $L_{r}(n, k)$. We have $[8$, p. 335, ex. 38]

$$
L_{r}(n, k)=(-1)^{n} \frac{n !}{k !}\left(\begin{array}{c}
n-r-1 \\
k-r-1
\end{array}\right) .
$$


(Observe that we have also a relation between the Blasiak's generalized Stirling numbers of the second kind and some generalized Lah numbers: see (8).)

Formula (20) can be written as

$$
\left(\begin{array}{c}
s n+r \\
p
\end{array}\right)=\frac{1}{p !} \sum_{k=0}^{p} k ! C_{r, s}(p, k)\left(\begin{array}{l}
n \\
k
\end{array}\right)
$$

which gives us the binomial coefficient $\left(\begin{array}{c}s n+r \\ p\end{array}\right)$ written as a linear combination of the binomial coefficients $\left(\begin{array}{l}n \\ k\end{array}\right), k=0,1, \ldots, p$. (Again we see that (24) has the same flavor of (15).) Note that (14) contains (15) (with integer $p>1$ ) which is not contained in (24). On the other hand, formula (24) in the case $s \neq 1, r \neq 0$, is not contained in (14). In fact, we have not any interesting intersection in both results, which reminds us that, despite the shared flavor of both objects, they appear in different directions. Some examples from (24) are the following

$$
\begin{aligned}
\left(\begin{array}{c}
2 n+1 \\
3
\end{array}\right) & =\left(\begin{array}{l}
n \\
1
\end{array}\right)+8\left(\begin{array}{l}
n \\
2
\end{array}\right)+8\left(\begin{array}{l}
n \\
3
\end{array}\right) \\
\left(\begin{array}{c}
2 n+1 \\
4
\end{array}\right) & =5\left(\begin{array}{l}
n \\
2
\end{array}\right)+20\left(\begin{array}{l}
n \\
3
\end{array}\right)+16\left(\begin{array}{l}
n \\
4
\end{array}\right), \\
\left(\begin{array}{c}
3 n+5 \\
4
\end{array}\right) & =5+65\left(\begin{array}{l}
n \\
1
\end{array}\right)+195\left(\begin{array}{l}
n \\
2
\end{array}\right)+216\left(\begin{array}{l}
n \\
3
\end{array}\right)+81\left(\begin{array}{l}
n \\
4
\end{array}\right) .
\end{aligned}
$$

\section{The main results}

In the following proposition we consider a version of (4) (and give a simple induction argument to prove it). This result (25), together with the corresponding formula (28) from proposition 3 , are the starting point of the results we will obtain in the remaining sections.

Proposition 2. The following formula holds

$$
\prod_{j=1}^{p}\left(\begin{array}{c}
n+(j-1)(r-s) \\
s
\end{array}\right)=\frac{1}{(s !)^{p}} \sum_{k=s}^{p s} k ! S_{r, s}(p, k)\left(\begin{array}{l}
n \\
k
\end{array}\right) .
$$

Proof. By induction on $p$. For $p=1$ formula (25) is trivial. Suppose it is true for a given $p \in \mathbb{N}$. Then, by using (7) and the induction hypothesis we have

$$
\begin{aligned}
& \frac{1}{(s !)^{p+1}} \sum_{k=s}^{p s+s} k ! S_{r, s}(p+1, k)\left(\begin{array}{l}
n \\
k
\end{array}\right) \\
= & \frac{1}{(s !)^{p+1}} \sum_{k=s}^{p s+s} k ! \sum_{i=0}^{s}\left(\begin{array}{c}
p(r-s)+k+i-s \\
i
\end{array}\right) \frac{s !}{(s-i) !} S_{r, s}(p, k+i-s)\left(\begin{array}{l}
n \\
k
\end{array}\right)
\end{aligned}
$$




$$
\begin{aligned}
& =\frac{1}{(s !)^{p}} \sum_{k=s}^{p s+s} \sum_{i=0}^{s}\left(\begin{array}{c}
p(r-s)+k-i \\
s-i
\end{array}\right) \frac{k !}{i !} S_{r, s}(p, k-i)\left(\begin{array}{l}
n \\
k
\end{array}\right) \\
& =\frac{1}{(s !)^{p}} \sum_{k=s}^{p s} \sum_{i=0}^{s}\left(\begin{array}{c}
p(r-s)+k \\
s-i
\end{array}\right) \frac{(i+k) !}{i !} S_{r, s}(p, k)\left(\begin{array}{c}
n \\
i+k
\end{array}\right) \\
& =\frac{1}{(s !)^{p}} \sum_{k=s}^{p s} k ! S_{r, s}(p, k)\left(\begin{array}{c}
n \\
k
\end{array}\right) \sum_{i=0}^{s}\left(\begin{array}{c}
p(r-s)+k \\
s-i
\end{array}\right)\left(\begin{array}{c}
n-k \\
i
\end{array}\right) \\
& =\left(\prod_{j=1}^{p}\left(\begin{array}{c}
n+(j-1)(r-s) \\
s
\end{array}\right)\right)\left(\begin{array}{c}
n+p(r-s) \\
s
\end{array}\right) \\
& =\prod_{j=1}^{p+1}\left(\begin{array}{c}
n+(j-1)(r-s) \\
s
\end{array}\right),
\end{aligned}
$$

as expected.

From expression (25), we have at once the corresponding expression of the partial sums of the sequence $\left(\begin{array}{l}n \\ r\end{array}\right)^{p}$ in terms of binomial coefficients. In fact, by using that

$$
\left(\begin{array}{c}
n \\
r_{1}
\end{array}\right) *\left(\begin{array}{c}
n \\
r_{2}
\end{array}\right)=\left(\begin{array}{c}
n+1 \\
r_{1}+r_{2}+1
\end{array}\right)
$$

we have for $l \in \mathbb{N}$

$$
\left(\begin{array}{l}
n \\
r
\end{array}\right)^{p} *^{l} 1=\frac{1}{(r !)^{p}} \sum_{j=r}^{p r} j ! S_{r, r}(p, j)\left(\begin{array}{c}
n+l \\
j+l
\end{array}\right) .
$$

However, we are interested in expressions of the partial sums $\left(\begin{array}{l}n \\ r\end{array}\right)^{p} *^{l} 1$ as linear combinations of the binomials $\left(\begin{array}{l}n \\ s\end{array}\right),\left(\begin{array}{c}n \\ s+1\end{array}\right),\left(\begin{array}{c}n \\ s+2\end{array}\right), \ldots$, since we want to study the number arrays formed by the corresponding coefficients of these expressions.

Proposition 3. For $k \geqslant p r-s+1$ we have

$$
\left(\prod_{j=1}^{p}\left(\begin{array}{c}
n+(j-1)(r-s) \\
s
\end{array}\right)\right) *^{k-p r+s-1} 1=\sum_{j=s}^{k-p(r-s)+s-1} a_{k, j}^{(r, s)}\left(\begin{array}{l}
n \\
j
\end{array}\right) .
$$

where the coefficients $a_{k, j}^{(r, s)}$ (for $s \leqslant j \leqslant k-p(r-s)+s-1$ ) are given by

$$
a_{k, j}^{(r, s)}=\frac{1}{(s !)^{p}} \sum_{i=s}^{j}\left(\begin{array}{c}
k-p r+s-1 \\
j-i
\end{array}\right) i ! S_{r, s}(p, i) .
$$

Proof. We proceed by induction on $k$. The case $k=p r-s+1$ is (25). Suppose the result is valid for a given $k \geqslant p r-s+1$. That is, suppose that

$$
\left(\prod_{j=1}^{p}\left(\begin{array}{c}
n+(j-1)(r-s) \\
s
\end{array}\right)\right) *^{k-p r+s-1} 1
$$




$$
=\frac{1}{(s !)^{p}} \sum_{j=s}^{k-p(r-s)+s-1} \sum_{i=s}^{j}\left(\begin{array}{c}
k-p r+s-1 \\
j-i
\end{array}\right) i ! S_{r, s}(p, i)\left(\begin{array}{l}
n \\
j
\end{array}\right) .
$$

By taking the convolution in both sides of (30) with 1 we obtain that

$$
\begin{aligned}
& \left(\prod_{j=1}^{p}\left(\begin{array}{c}
n+(j-1)(r-s) \\
s
\end{array}\right)\right) *^{k+1-p r+s-1} 1 \\
= & \frac{1}{(s !)^{p}} \sum_{j=s}^{k-p(r-s)+s-1} \sum_{i=s}^{j}\left(\begin{array}{c}
k-p r+s-1 \\
j-i
\end{array}\right) i ! S_{r, s}(p, i)\left(\begin{array}{c}
n+1 \\
j+1
\end{array}\right) .
\end{aligned}
$$

Thus, we need to prove that

$$
\begin{gathered}
\sum_{j=s}^{k-p(r-s)+s-1} \sum_{i=s}^{j}\left(\begin{array}{c}
k-p r+s-1 \\
j-i
\end{array}\right) i ! S_{r, s}(p, i)\left(\begin{array}{c}
n+1 \\
j+1
\end{array}\right) \\
=\sum_{j=s}^{k+1-p(r-s)+s-1} \sum_{i=s}^{j}\left(\begin{array}{c}
k+1-p r+s-1 \\
j-i
\end{array}\right) i ! S_{r, s}(p, i)\left(\begin{array}{l}
n \\
j
\end{array}\right),
\end{gathered}
$$

and this is an easy exercise left to the reader.

We form the $(r, s, p)$-arrays with the coefficients $a_{k, j}^{(r, s)}$ ( $k$ for rows and $j$ for columns). The first $(p r-s)$ rows are cancelled, so the first non-cancelled row is the $(p r-s+1)$ th one, which contains the coefficients of the right-hand side of (25) (corresponding to $k=p r-s+1$ of $(28))$. The first $(s-1)$ columns are cancelled, and the corresponding columns of the $k$-th row goes from the $s$-th column up to the $(k-p(r-s)+s-1)$ th column (with zeros in the subsequent places). Since the coefficients $a_{k, j}^{(r, s)}$ have the Pascal's triangle property $a_{k, j}^{(r, s)}+a_{k, j+1}^{(r, s)}=a_{k+1, j+1}^{(r, s)}$, we can also form the $(r, s, p)$-arrays beginning with the first non-cancelled row, and filling-out the array with the Pascal's triangle recurrence.

For example, in the case $r=s$ (formula (18)), if we set $k=s(p-1)+2$, we can write

$$
\sum_{i=0}^{n}\left(\begin{array}{l}
i \\
s
\end{array}\right)^{p}=\frac{1}{(s !)^{p}} \sum_{j=s}^{p s+1}(j-1) !\left(j S_{s, s}(p, j)+S_{s, s}(p, j-1)\right)\left(\begin{array}{l}
n \\
j
\end{array}\right) .
$$

(The case $s=1$ of (33) is the well-known formula $\sum_{i=0}^{n} i^{p}=\sum_{j=1}^{p+1}(j-1) ! S(p+1, j)\left(\begin{array}{l}n \\ j\end{array}\right)$ for sums of powers of integers.) In the case $r=s$ (18), if we set $p=1$ we obtain that (for $k \geqslant 1)$

$$
\left(\begin{array}{l}
n \\
s
\end{array}\right) *^{k-1} 1=\sum_{j=0}^{k-1}\left(\begin{array}{c}
k-1 \\
j
\end{array}\right)\left(\begin{array}{c}
n \\
j+s
\end{array}\right)
$$

which gives us the partial sums of the sequence $\left(\begin{array}{l}n \\ s\end{array}\right)$ in terms of the binomials $\left(\begin{array}{c}n \\ j+s\end{array}\right), j=$ $0,1, \ldots, k-1$. For example, $\sum_{i=0}^{n}\left(\begin{array}{l}i \\ s\end{array}\right)=\left(\begin{array}{l}n \\ s\end{array}\right)+\left(\begin{array}{c}n \\ s+1\end{array}\right), \sum_{j=0}^{n} \sum_{i=0}^{j}\left(\begin{array}{l}i \\ s\end{array}\right)=\left(\begin{array}{l}n \\ s\end{array}\right)+2\left(\begin{array}{c}n \\ s+1\end{array}\right)+\left(\begin{array}{c}n \\ s+2\end{array}\right)$, 
$\sum_{k=0}^{n} \sum_{j=0}^{k} \sum_{i=0}^{j}\left(\begin{array}{l}i \\ s\end{array}\right)=\left(\begin{array}{c}n \\ s\end{array}\right)+3\left(\begin{array}{c}n \\ s+1\end{array}\right)+3\left(\begin{array}{c}n \\ s+2\end{array}\right)+\left(\begin{array}{c}n \\ s+3\end{array}\right)$, and so on. That is, the $(s, s, 1)$-array is a "right-shifted Pascal's triangle", with its first column (the constant sequence 1) in the $s$-th column of the array.

In the case $r=2 s$ we can use (8) to write (28) as

$$
\begin{aligned}
& \left(\prod_{j=1}^{p}\left(\begin{array}{c}
n+s(j-1) \\
s
\end{array}\right)\right) *^{k-2 p s+s-1} 1 \\
= & \frac{(s p) !}{(s !)^{p}} \sum_{j=s}^{k-s(p-1)-1} \sum_{i=s}^{j}\left(\begin{array}{c}
k-2 p s+s-1 \\
j-i
\end{array}\right)\left(\begin{array}{c}
s(p-1) \\
i-s
\end{array}\right)\left(\begin{array}{l}
n \\
j
\end{array}\right) .
\end{aligned}
$$

or

$$
\left(\prod_{j=1}^{p}\left(\begin{array}{c}
n+s(j-1) \\
s
\end{array}\right)\right) *^{k-2 p s+s-1} 1=\frac{(s p) !}{(s !)^{p}} \sum_{j=s}^{k-s(p-1)-1}\left(\begin{array}{c}
k-p s-1 \\
j-s
\end{array}\right)\left(\begin{array}{l}
n \\
j
\end{array}\right)
$$

where $k \geqslant 2 p s-s+1$. Note then that the $(2 s, s, p)$-array is a "piece of Pascal's triangle multiplied by $\frac{(s p) !}{(s !)^{p} "}$ : the first non-cancelled row of the array (the $(2 p s-s+1)$-th row) contains the $s(p-1)$-th row of Pascal's triangle (multiplied by the factor $\left.\frac{(s p) !}{(s !)^{p}}\right)$; the first non-cancelled column of the array (the $s$-th column) contains the first column of Pascal's triangle (multiplied by the factor $\frac{(s p) !}{(s !)^{p}}$. That is, the $s$-th column of the array is the constant sequence $\frac{(s p) !}{(s !)^{p}}$. The subsequent rows and columns of the array are the corresponding subsequent rows and columns of Pascal's triangle multiplied by $\frac{(s p) !}{(s !)^{p}}$. See for example the $(2,1,3)$-array below, which is a Pascal's triangle beginning in row $s(p-1)=2$ and multiplied by $\frac{(s p) !}{(s !)^{p}}=6$, and the $(4,2,3)$-array which is a Pascal's triangle beginning in

\begin{tabular}{|c|c|c|c|c|c|c|c|c|c|c|c|c|c|c|c|c|c|}
\hline \multicolumn{8}{|c|}{$(2,1,3)$-array, $k \geqslant 6,1 \leqslant j \leqslant k-3$} & \multicolumn{10}{|c|}{$(2,2,3)$-array, $k \geqslant 5,2 \leqslant j \leqslant k+1$} \\
\hline$*$ & $*$ & $*$ & $*$ & $*$ & $*$ & $*$ & $*$ & $*$ & $*$ & * & * & $*$ & $*$ & * & $*$ & $*$ & $*$ \\
\hline$*$ & $*$ & $*$ & $*$ & $*$ & $*$ & $*$ & $*$ & $*$ & * & $*$ & $*$ & $*$ & $*$ & * & $*$ & $*$ & $*$ \\
\hline$*$ & $*$ & $*$ & $*$ & * & $*$ & $*$ & $*$ & $*$ & $*$ & $*$ & $*$ & $*$ & $*$ & $*$ & $*$ & $*$ & $*$ \\
\hline$*$ & $*$ & $*$ & $*$ & $*$ & $*$ & $*$ & $*$ & $*$ & $*$ & $*$ & $*$ & $*$ & $*$ & $*$ & $*$ & $*$ & $*$ \\
\hline$*$ & $*$ & * & $*$ & * & * & $*$ & $*$ & * & 1 & 24 & 114 & 180 & 90 & 0 & 0 & 0 & $\cdots$ \\
\hline 6 & 12 & 6 & 0 & U & 0 & 0 & $\cdots$ & $*$ & 1 & 25 & 138 & 294 & 270 & 90 & 0 & 0 & \\
\hline 6 & 18 & 18 & 6 & 0 & 0 & 0 & & $*$ & 1 & 26 & 163 & 432 & 564 & 360 & 90 & 0 & \\
\hline 6 & 24 & 36 & 24 & 6 & 0 & 0 & & . & 1 & 7 & 10 & - & (1) & ת & - & مת & \\
\hline 6 & 30 & 60 & 60 & 30 & 6 & 0 & $\ldots$ & $*$ & 1 & 27 & 189 & 595 & 990 & 924 & 450 & 90 & • \\
\hline : & & & $\therefore$ & & & : & & $*$ & : & & & $\vdots$ & & & $\vdots$ & & \\
\hline
\end{tabular}
row $s(p-1)=4$ and multiplied by $\frac{(s p) !}{(s !)^{p}}=90$.

Some $(r, s, p)$-arrays are the following: 


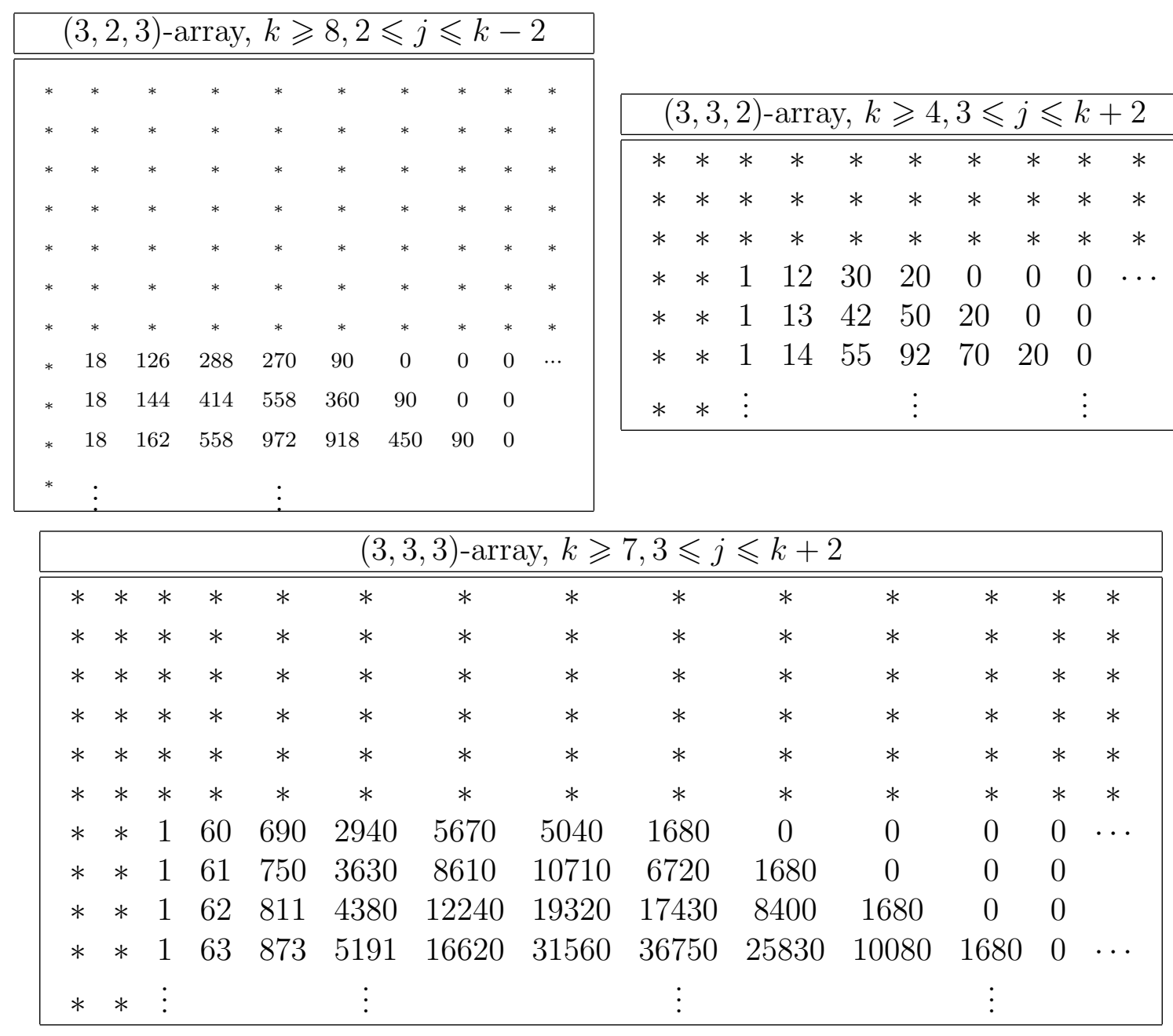

\begin{tabular}{|c|c|c|c|c|c|c|c|}
\hline \multicolumn{8}{|c|}{$(4,1,3)$-array, $k \geqslant 12,1 \leqslant j \leqslant k-9$} \\
\hline$*$ & $*$ & $*$ & $*$ & $*$ & $*$ & $*$ & $*$ \\
\hline$*$ & $*$ & $*$ & $*$ & $*$ & $*$ & $*$ & $*$ \\
\hline$*$ & $*$ & $*$ & $*$ & $*$ & $*$ & $*$ & $*$ \\
\hline$*$ & $*$ & $*$ & $*$ & $*$ & $*$ & $*$ & $*$ \\
\hline$*$ & $*$ & $*$ & $*$ & $*$ & $*$ & $*$ & $*$ \\
\hline$*$ & $*$ & $*$ & $*$ & $*$ & $*$ & $*$ & $*$ \\
\hline$*$ & $*$ & $*$ & $*$ & $*$ & $*$ & $*$ & $*$ \\
\hline$*$ & $*$ & $*$ & $*$ & $*$ & $*$ & $*$ & $*$ \\
\hline$*$ & $*$ & $*$ & $*$ & $*$ & $*$ & $*$ & $*$ \\
\hline$*$ & $*$ & $*$ & $*$ & $*$ & $*$ & * & $*$ \\
\hline$*$ & $*$ & $*$ & $*$ & $*$ & $*$ & $*$ & $*$ \\
\hline 28 & 24 & 6 & 0 & 0 & 0 & 0 & $\ldots$ \\
\hline 28 & 52 & 30 & 6 & 0 & 0 & 0 & \\
\hline 28 & 80 & 82 & 36 & 6 & 0 & 0 & \\
\hline 28 & 108 & 162 & 118 & 42 & 6 & 0 & $\ldots$ \\
\hline$\vdots$ & & & : & & & $\vdots$ & \\
\hline
\end{tabular}

\begin{tabular}{|ccccccccccc|}
\hline \multicolumn{1}{cc}{$(4,2,3)$-array, $k \geqslant 11,2 \leqslant j \leqslant$} & \multicolumn{1}{c|}{$(1,5$} \\
\hline \hline$*$ & $*$ & $*$ & $*$ & $*$ & $*$ & $*$ & $*$ & $*$ & $*$ \\
$*$ & $*$ & $*$ & $*$ & $*$ & $*$ & $*$ & $*$ & $*$ & $*$ \\
$*$ & $*$ & $*$ & $*$ & $*$ & $*$ & $*$ & $*$ & $*$ & $*$ \\
$*$ & $*$ & $*$ & $*$ & $*$ & $*$ & $*$ & $*$ & $*$ & $*$ \\
$*$ & $*$ & $*$ & $*$ & $*$ & $*$ & $*$ & $*$ & $*$ & $*$ \\
$*$ & $*$ & $*$ & $*$ & $*$ & $*$ & $*$ & $*$ & $*$ & $*$ \\
$*$ & $*$ & $*$ & $*$ & $*$ & $*$ & $*$ & $*$ & $*$ & $*$ \\
$*$ & $*$ & $*$ & $*$ & $*$ & $*$ & $*$ & $*$ & $*$ & $*$ \\
$*$ & $*$ & $*$ & $*$ & $*$ & $*$ & $*$ & $*$ & $*$ & $*$ \\
$*$ & $*$ & $*$ & $*$ & $*$ & $*$ & $*$ & $*$ & $*$ & $*$ \\
$*$ & 90 & 360 & 540 & 360 & 90 & 0 & 0 & 0 & $\cdots$ \\
$*$ & 90 & 450 & 900 & 900 & 450 & 90 & 0 & 0 & \\
$*$ & 90 & 540 & 1350 & 1800 & 1350 & 540 & 90 & 0 & \\
$*$ & $\vdots$ & & & $\vdots$ & & & $\vdots$ & & \\
\hline
\end{tabular}




\begin{tabular}{|cccccccccc|}
\hline \multicolumn{1}{|c|}{$(4,3,2)$-array, $k \geqslant 6,3 \leqslant j \leqslant k$} \\
\hline \hline$*$ & $*$ & $*$ & $*$ & $*$ & $*$ & $*$ & $*$ & $*$ & $*$ \\
$*$ & $*$ & $*$ & $*$ & $*$ & $*$ & $*$ & $*$ & $*$ & $*$ \\
$*$ & $*$ & $*$ & $*$ & $*$ & $*$ & $*$ & $*$ & $*$ & $*$ \\
$*$ & $*$ & $*$ & $*$ & $*$ & $*$ & $*$ & $*$ & $*$ & $*$ \\
$*$ & $*$ & $*$ & $*$ & $*$ & $*$ & $*$ & $*$ & $*$ & $*$ \\
$*$ & $*$ & 4 & 24 & 40 & 20 & 0 & 0 & 0 & $\cdots$ \\
$*$ & $*$ & 4 & 28 & 64 & 60 & 20 & 0 & 0 & \\
$*$ & $*$ & 4 & 32 & 92 & 124 & 80 & 20 & 0 & \\
$*$ & $*$ & $\vdots$ & & & $\vdots$ & & & & \\
\hline
\end{tabular}

\begin{tabular}{|c|c|c|c|c|c|c|c|c|c|c|c|}
\hline \multicolumn{12}{|c|}{$(4,4,2)$-array, $k \geqslant 5,4 \leqslant j \leqslant k+3$} \\
\hline$*$ & $*$ & * & $*$ & $*$ & $*$ & $*$ & $*$ & $*$ & $*$ & $*$ & $*$ \\
\hline$*$ & $*$ & * & $*$ & $*$ & * & * & * & * & $*$ & $*$ & $*$ \\
\hline$*$ & $*$ & * & $*$ & $*$ & * & $*$ & * & * & $*$ & $*$ & $*$ \\
\hline$*$ & $*$ & $*$ & $*$ & $*$ & $*$ & $*$ & * & $*$ & $*$ & $*$ & $*$ \\
\hline$*$ & $*$ & $*$ & 1 & 20 & 90 & 140 & 70 & 0 & 0 & 0 & $\ldots$ \\
\hline * & $*$ & $*$ & 1 & 21 & 110 & 230 & 210 & 70 & 0 & 0 & \\
\hline$*$ & $*$ & $*$ & 1 & 22 & 131 & 340 & 440 & 280 & 70 & 0 & \\
\hline * & * & & & & & . & & & . & & \\
\hline
\end{tabular}

Some examples from (30) are shown in the following table.

\begin{tabular}{|c|c|c|}
\hline Array & $k$ & Sequence (30) \\
\hline$(2,1,3)$ & 7 & $\sum_{i=1}^{n} i(i+1)(i+2)=6\left(\begin{array}{l}n \\
1\end{array}\right)+18\left(\begin{array}{l}n \\
2\end{array}\right)+18\left(\begin{array}{l}n \\
3\end{array}\right)+6\left(\begin{array}{l}n \\
4\end{array}\right)$ \\
\hline$(2,2,3)$ & 6 & $\sum_{i=1}^{n}\left(\begin{array}{l}i \\
2\end{array}\right)^{3}=\left(\begin{array}{l}n \\
2\end{array}\right)+25\left(\begin{array}{l}n \\
3\end{array}\right)+138\left(\begin{array}{l}n \\
4\end{array}\right)+294\left(\begin{array}{l}n \\
5\end{array}\right)+270\left(\begin{array}{l}n \\
6\end{array}\right)+90\left(\begin{array}{l}n \\
7\end{array}\right)$ \\
\hline$(3,2,3)$ & 8 & $\left(\begin{array}{l}n \\
2\end{array}\right)\left(\begin{array}{c}n+1 \\
2\end{array}\right)\left(\begin{array}{c}n+2 \\
2\end{array}\right)=18\left(\begin{array}{l}n \\
2\end{array}\right)+126\left(\begin{array}{l}n \\
3\end{array}\right)+288\left(\begin{array}{l}n \\
4\end{array}\right)+270\left(\begin{array}{l}n \\
5\end{array}\right)+90\left(\begin{array}{c}n \\
6\end{array}\right)$ \\
\hline$(3,3,2)$ & 5 & $\sum_{i=1}^{n}\left(\begin{array}{l}i \\
3\end{array}\right)^{2}=\left(\begin{array}{l}n \\
3\end{array}\right)+13\left(\begin{array}{l}n \\
4\end{array}\right)+42\left(\begin{array}{l}n \\
5\end{array}\right)+50\left(\begin{array}{l}n \\
6\end{array}\right)+20\left(\begin{array}{l}n \\
7\end{array}\right)$ \\
\hline$(3,3,3)$ & 7 & $\left(\begin{array}{l}n \\
3\end{array}\right)^{3}=\left(\begin{array}{l}n \\
3\end{array}\right)+60\left(\begin{array}{l}n \\
4\end{array}\right)+690\left(\begin{array}{l}n \\
5\end{array}\right)+2940\left(\begin{array}{l}n \\
6\end{array}\right)+5670\left(\begin{array}{l}n \\
7\end{array}\right)+5040\left(\begin{array}{l}n \\
8\end{array}\right)+1680\left(\begin{array}{l}n \\
9\end{array}\right)$ \\
\hline$(4,1,3)$ & 12 & $n(n+3)(n+6)=28\left(\begin{array}{l}n \\
1\end{array}\right)+24\left(\begin{array}{l}n \\
2\end{array}\right)+6\left(\begin{array}{l}n \\
3\end{array}\right)$ \\
\hline$(4,2,3)$ & 12 & $\sum_{i=1}^{n}\left(\begin{array}{c}i+2 \\
2\end{array}\right)\left(\begin{array}{c}i+4 \\
2\end{array}\right)\left(\begin{array}{c}i+6 \\
2\end{array}\right)=90\left(\begin{array}{l}n \\
2\end{array}\right)+450\left(\begin{array}{l}n \\
3\end{array}\right)+900\left(\begin{array}{c}n \\
4\end{array}\right)+900\left(\begin{array}{l}n \\
5\end{array}\right)+450\left(\begin{array}{l}n \\
6\end{array}\right)+90\left(\begin{array}{c}n \\
7\end{array}\right)$ \\
\hline$(4,3,2)$ & 6 & $\left(\begin{array}{c}n \\
3\end{array}\right)\left(\begin{array}{c}n+1 \\
3\end{array}\right)=4\left(\begin{array}{l}n \\
3\end{array}\right)+24\left(\begin{array}{c}n \\
4\end{array}\right)+40\left(\begin{array}{l}n \\
5\end{array}\right)+20\left(\begin{array}{c}n \\
6\end{array}\right)$ \\
\hline$(4,4,2)$ & 6 & $\sum_{i=1}^{n}\left(\begin{array}{l}i \\
4\end{array}\right)^{2}=\left(\begin{array}{l}n \\
4\end{array}\right)+21\left(\begin{array}{l}n \\
5\end{array}\right)+110\left(\begin{array}{l}n \\
6\end{array}\right)+230\left(\begin{array}{l}n \\
7\end{array}\right)+210\left(\begin{array}{l}n \\
8\end{array}\right)+70\left(\begin{array}{l}n \\
9\end{array}\right)$ \\
\hline
\end{tabular}

Table 1: Examples of sequence (23). The coefficients come from k-th line of the (r,s,p)array.

\section{Some properties (rows, columns, falling diagonals)}

From the well-known properties of rows, columns and falling diagonals in Pascal's triangle, we state in this section the corresponding generalizations of them for our $(r, s, p)$-arrays. Most of the proofs of these facts are easy exercises left to the reader.

We begin by considering the rows of $(r, s, p)$-arrays.

\subsection{Rows}

We mention (leaving the proofs to the reader) the generalization of two famous properties of rows of Pascal's triangle: 
1. In a $(r, s, p)$-array, the sum of the elements of the $k$-th row $(k>p r-s+1)$ is equal to $2^{k-p r+s-1}$ times the sum of the elements of the $(p r-s+1)$-th row (the first non-cancelled row). That is, we have

$$
\sum_{j=s}^{k-p(r-s)+s-1} a_{k, j}^{(r, s)}=2^{k-p r+s-1} \sum_{j=s}^{p s} a_{p r-s+1, j}^{(r, s)},
$$

or more explicitly

$$
\sum_{j=s}^{k-p(r-s)+s-1} \sum_{i=s}^{j}\left(\begin{array}{c}
k-p r+s-1 \\
j-i
\end{array}\right) i ! S_{r, s}(p, i)=2^{k-p r+s-1} \sum_{j=s}^{p s} j ! S_{r, s}(p, j) .
$$

2. Making the row $k \geqslant p r-s+1$ a single number $N_{k}$ (the elements of the row being the digits, and carrying over when appear elements with more than one digit), this is equal to $11^{k-p r+s-1} N_{p r-s+1}$, where $N_{p r-s+1}$ is the single number corresponding to row $k=p r-s+1$.

In Table 2 we have some examples taken from the first rows of the $(4,3,2)$-array.

\begin{tabular}{|c|c|c|c|}
\hline $\begin{array}{l}\text { Beginning } \\
\text { in column } \\
j=3 \\
\text { and row } k\end{array}$ & The row is & $\begin{array}{l}\text { Sum of the } \\
\text { elements }\end{array}$ & $\begin{array}{l}\text { As a single } \\
\text { number }\end{array}$ \\
\hline \hline$k=6$ & $(4,24,40,20)$ & 88 & 6820 \\
\hline$k=7$ & $(4,28,64,60,20)$ & $176=2(88)$ & $75020=11(6820)$ \\
\hline$k=8$ & $(4,32,92,124,80,20)$ & $352=2^{2}(88)$ & $825220=11^{2}(6820)$ \\
\hline$\vdots$ & $\vdots$ & $\vdots$ & $\vdots$ \\
\hline
\end{tabular}

Table 2: Properties of rows in the (4,3,2)-array.

A more interesting question arises when one considers the sequence of alternating sums of rows of a $(r, s, p)$-array. In the case $r=s=1$, we proved that this sequence is $\left((-1)^{p}, 0,0,0, \ldots\right)$ (see formula $(26)$ in $\left.[24]\right)$. In general, for a $(r, s, p)$-array, we have only some partial answers, as we show next.

First to all note that for $k>p r-s+1$, the alternating row sums of the $(r, s, p)$-array are equal to 0 (easy proof left to the reader). The alternating sum of the elements of row $k=p r-s+1$ is equal to

$$
\alpha_{r, s, p}=\frac{1}{(s !)^{p}} \sum_{j=s}^{p s}(-1)^{j} j ! S_{r, s}(p, j) .
$$

Thus, (ignoring the cancelled rows) the alternating sums of rows in a $(r, s, p)$-array, are sequences of the form $\left(\alpha_{r, s, p}, 0,0,0, \ldots\right)$. Let us see some particular cases in which the term $\alpha_{r, s, p}$ has a simple closed formula. 
If $s=r$ we have $\alpha_{s, s, p}=(-1)^{p s}$. That is, we have

$$
\frac{1}{(s !)^{p}} \sum_{j=s}^{p s}(-1)^{j} j ! S_{s, s}(p, j)=(-1)^{p s} .
$$

In fact, proceeding by induction on $p$, we see at once that this formula is trivial if $p=1$. If it is true for a given $p \in \mathbb{N}$, then (according to the recurrence (7))

$$
\begin{aligned}
& \frac{1}{(s !)^{p+1}} \sum_{j=s}^{(p+1) s}(-1)^{j} j ! S_{s, s}(p+1, j) \\
= & \frac{1}{(s !)^{p+1}} \sum_{j=s}^{(p+1) s}(-1)^{j} j ! \sum_{i=0}^{s}\left(\begin{array}{c}
j+i-s \\
i
\end{array}\right) \frac{s !}{(s-i) !} S_{s, s}(p, j+i-s) \\
= & \frac{(-1)^{s}}{(s !)^{p}} \sum_{j=s}^{p s} \sum_{i=0}^{s} \frac{(-1)^{j-i}(j-i+s) ! j !}{(s-i) ! i !(j-i) !} S_{s, s}(p, j) \\
= & \frac{(-1)^{s}}{(s !)^{p}} \sum_{j=s}^{p s}(-1)^{j} j ! \sum_{i=0}^{s}(-1)^{i}\left(\begin{array}{c}
s \\
i
\end{array}\right)\left(\begin{array}{c}
j-i+s \\
s
\end{array}\right) S_{s, s}(p, j) .
\end{aligned}
$$

But we have $\sum_{i=0}^{s}(-1)^{i}\left(\begin{array}{l}s \\ i\end{array}\right)\left(\begin{array}{c}j-i+s \\ s\end{array}\right)=1$ (see identity (53) below, or identity (1.83) in [14]). Thus, by using the induction hypothesis we have

$$
\begin{aligned}
\frac{1}{(s !)^{p+1}} \sum_{j=s}^{(p+1) s}(-1)^{j} j ! S_{s, s}(p+1, j) & =\frac{(-1)^{s}}{(s !)^{p}} \sum_{j=s}^{p s}(-1)^{j} j ! S_{s, s}(p, j) \\
& =(-1)^{s}(-1)^{p s}=(-1)^{(p+1) s}
\end{aligned}
$$

as expected. That is, the alternating sum of rows in the $(s, s, p)$-arrays is the sequence $\left((-1)^{p s}, 0,0,0, \ldots\right)$.

Other interesting particular case is when $s=1$. We claim that

$$
\alpha_{r, 1, p}=\sum_{j=1}^{p}(-1)^{j} j ! S_{r, 1}(p, j)=\prod_{i=1}^{p}((r-1) i-r) .
$$

The case $p=1$ is obvious. If this formula is true for a given $p \in \mathbb{N}$, we have (by using the recurrence $(7)$ )

$$
\begin{aligned}
& \sum_{j=1}^{p+1}(-1)^{j} j ! S_{r, 1}(p+1, j) \\
= & \sum_{j=1}^{p+1}(-1)^{j} j !\left(S_{r, 1}(p, j-1)+(p(r-1)+j) S_{r, 1}(p, j)\right)
\end{aligned}
$$




$$
\begin{aligned}
= & \sum_{j=1}^{p}(-1)^{j+1} j !(j+1) S_{r, 1}(p, j)+p(r-1) \sum_{j=1}^{p}(-1)^{j} j ! S_{r, 1}(p, j) \\
& +\sum_{j=1}^{p}(-1)^{j} j ! j S_{r, 1}(p, j) \\
= & (p(r-1)-1) \sum_{j=1}^{p}(-1)^{j} j ! S_{r, 1}(p, j) \\
= & (p(r-1)-1) \prod_{i=1}^{p}((r-1) i-r)=\prod_{i=1}^{p+1}((r-1) i-r) .
\end{aligned}
$$

as expected. Some examples (within the particular case $s=1$ we are considering) are the following:

- If $r=1$ we have $\alpha_{1,1, p}=(-1)^{p}$ (as we obtained before).

- If $r=2$ we have $\alpha_{2,1, p}=\prod_{i=1}^{p}(i-2)$, so $\alpha_{2,1, p}=-1$ if $p=1$ and $\alpha_{2,1, p}=0$ if $p>1$. That is, the alternating sums of rows in the $(2,1, p)$-arrays is the sequence $(-1,0,0,0, \ldots)$ when $p=1$, and the constant sequence 0 when $p>1$.

- For $r>2$, we see from (40) that $\alpha_{r, 1, p}$ is the $(r-1)$-fold factorial:

- With $r=3$ we have the $p$-sequence

$$
\left(\alpha_{3,1, p}\right)_{p \in \mathbb{N}}=\left(\prod_{i=1}^{p}(2 i-3)\right)_{p \in \mathbb{N}}=(-1,-1,-3,-15, \ldots)
$$

formed by the (negatives of the) double factorials $(2 p-3)$ !! (see [25, A001147] for $(2 p-1) ! !)$. For example, the alternating sums of rows in the $(3,1,2)$-array is the sequence $(-1,0,0,0, \ldots)$, and the alternating sums of rows in the $(3,1,3)$ array is the sequence $(-3,0,0,0, \ldots)$.

- With $r=4$ we have the $p$-sequence

$$
\left(\alpha_{4,1, p}\right)_{p \in \mathbb{N}}=\left(\prod_{i=1}^{p}(3 i-4)\right)_{p \in \mathbb{N}}=(-1,-2,-10,-80, \ldots)
$$

formed by the (negatives of the) triple factorials $(3 p-4)$ !!! (see [25, A008544] for $(3 p-1) ! ! !)$. For example, the alternating sums of rows in the $(4,1,2)$ array is the sequence $(-2,0,0,0, \ldots)$, and the alternating sums of rows in the $(4,1,3)$-array is the sequence $(-10,0,0,0, \ldots)$.

- With $r=5$ we have the $p$-sequence

$$
\left(\alpha_{5,1, p}\right)_{p \in \mathbb{N}}=\left(\prod_{i=1}^{p}(4 i-5)\right)_{p \in \mathbb{N}}=(-1,-3,-21,-231, \ldots)
$$


of the (negatives of the) quadruple factorials $(4 p-5)$ !!!! (see [25, A008545] for $(4 p-1) ! ! ! !)$. For example, the alternating sums of rows in the $(5,1,2)$ array is the sequence $(-3,0,0,0, \ldots)$, and the alternating sums of rows in the $(5,1,3)$-array is the sequence $(-21,0,0,0, \ldots)$.

\subsection{Columns}

According to (29), the $s$-th column (first non-cancelled column) in the $(r, s, p)$-array is the (constant) sequence

$$
a_{k, s}^{(r, s)}=\frac{1}{(s !)^{p}} \sum_{i=s}^{s}\left(\begin{array}{c}
k-p r+s-1 \\
s-i
\end{array}\right) i ! S_{r, s}(p, i)=\frac{S_{r, s}(p, s)}{(s !)^{p-1}} .
$$

For $j \geqslant s$, the $j$-th column is

$$
\left(\frac{1}{(s !)^{p}} \sum_{i=s}^{j}\left(\begin{array}{l}
l-1 \\
j-i
\end{array}\right) i ! S_{r, s}(p, i)\right)_{l \in \mathbb{N}}
$$

If $j \geqslant p s$, we can shift the corresponding sequence in order to show only the non-zero terms:

$$
\left(\frac{1}{(s !)^{p}} \sum_{i=s}^{j}\left(\begin{array}{c}
l+j-p s-1 \\
j-i
\end{array}\right) i ! S_{r, s}(p, i)\right)_{l \in \mathbb{N}} .
$$

For $j>p s$, we have the hockey-stick property (proof left to the reader)

$$
\sum_{l=1}^{m} \sum_{i=s}^{j}\left(\begin{array}{c}
l+j-p s-1 \\
j-i
\end{array}\right) i ! S_{r, s}(p, i)=\sum_{i=s}^{j+1}\left(\begin{array}{c}
m+j-p s \\
j+1-i
\end{array}\right) i ! S_{r, s}(p, i) .
$$

In general we have for $j>p s$ and $t, m \in \mathbb{N}$,

$$
\sum_{i_{t}=1}^{m} \cdots \sum_{i_{2}=1}^{i_{3}} \sum_{i_{1}=1}^{i_{2}} \sum_{i=s}^{j}\left(\begin{array}{c}
i_{1}+j-p s-1 \\
j-i
\end{array}\right) i ! S_{r, s}(p, i)=\sum_{i=s}^{j+t}\left(\begin{array}{c}
m+t-1+j-p s \\
j+1-t
\end{array}\right) i ! S_{r, s}(p, i) .
$$

\subsection{Falling Diagonals}

We consider first falling diagonals of the $(r, s, p)$-array, beginning in row $k \geqslant p r-s+1$ and column $j=s$. According to (29) these diagonals are the sequences (where $n \in \mathbb{N}$ )

$$
a_{k+n-1, s+n-1}^{(r, s)}=\frac{1}{(s !)^{p}} \sum_{i=s}^{p s}\left(\begin{array}{c}
k+n-p r+s-2 \\
s+n-1-i
\end{array}\right) i ! S_{r, s}(p, i) .
$$


In particular, observe that the falling diagonal beginning in row $k=p r+1$ is

$$
a_{p r+n, s+n-1}^{(r, s)}=\frac{1}{(s !)^{p}} \sum_{i=s}^{p s}\left(\begin{array}{c}
n+s-1 \\
s+n-1-i
\end{array}\right) i ! S_{r, s}(p, i)=\frac{1}{(s !)^{p}} \sum_{i=s}^{p s}\left(\begin{array}{c}
n+s-1 \\
i
\end{array}\right) i ! S_{r, s}(p, i) .
$$

That is, the falling diagonal beginning in row $k=p r+1$ and column $j=s$ in the $(r, s, p)$-array is, according to $(25)$, the sequence

$$
\left(\prod_{j=1}^{p}\left(\begin{array}{c}
n+s-1+(j-1)(r-s) \\
s
\end{array}\right)\right)_{n \in \mathbb{N}}
$$

(the original sequence involved in the array). For example, let us consider the $(3,2,3)$ array: the starting point of this array is the sequence $\left(\left(\begin{array}{c}n+1 \\ 2\end{array}\right)\left(\begin{array}{c}n+2 \\ 2\end{array}\right)\left(\begin{array}{c}n+3 \\ 2\end{array}\right)\right)_{n \in \mathbb{N}}$. When writing this sequence in terms of the binomials $\left(\begin{array}{l}n \\ 2\end{array}\right),\left(\begin{array}{l}n \\ 3\end{array}\right), \ldots,\left(\begin{array}{l}n \\ 6\end{array}\right)$, we obtain the coefficients $18,126,288,270,90$ (which form the "first" -non-cancelled- row of the array). Then we form the $(3,2,3)$-array by using the Pascal's triangle recurrence. In this array, the falling diagonal beginning in row $k=p r+1=10$ and column $j=s=2$ is precisely the original sequence $\left(\left(\begin{array}{c}n+1 \\ 2\end{array}\right)\left(\begin{array}{c}n+2 \\ 2\end{array}\right)\left(\begin{array}{c}n+3 \\ 2\end{array}\right)\right)_{n \in \mathbb{N}}=(18,180,900,3150, \ldots)$.

From the falling diagonal beginning in row $k=p r+1$, the subsequent diagonals can be obtained as the partial sums of the previous diagonal. That is, we have the hockey-stick property (proof left to the reader)

$$
\sum_{i_{l}=1}^{n} \cdots \sum_{i_{2}=1}^{i_{3}} \sum_{i_{1}=1}^{i_{2}} \prod_{j=1}^{p}\left(\begin{array}{c}
i_{1}+s-1+(j-1)(r-s) \\
s
\end{array}\right)=\frac{1}{(s !)^{p}} \sum_{i=s}^{p s}\left(\begin{array}{c}
n+s+l-1 \\
i+l
\end{array}\right) i ! S_{r, s}(p, i),
$$

where $l \in \mathbb{N}$. In fact, observe that the falling diagonal beginning in row $k=p r$ (and column $j=s$ ) is

$$
\begin{aligned}
a_{p r+n-1, s+n-1}^{(r, s)} & =\frac{1}{(s !)^{p}} \sum_{i=s}^{p s}\left(\begin{array}{c}
n+s-2 \\
s+n-1-i
\end{array}\right) i ! S_{r, s}(p, i) \\
& =\frac{1}{(s !)^{p}} \sum_{i=s}^{p s}\left(\begin{array}{c}
n+s-2 \\
i-1
\end{array}\right) i ! S_{r, s}(p, i) \\
& =\frac{1}{(s !)^{p}} \sum_{i=s}^{p s}\left(\left(\begin{array}{c}
n+s-1 \\
i
\end{array}\right)-\left(\begin{array}{c}
n+s-2 \\
i
\end{array}\right)\right) i ! S_{r, s}(p, i) \\
& =\frac{1}{(s !)^{p}} \sum_{i=s}^{p s}\left(\begin{array}{c}
n+s-1 \\
i
\end{array}\right) i ! S_{r, s}(p, i)-\frac{1}{(s !)^{p}} \sum_{i=s}^{p s}\left(\begin{array}{c}
n+s-2 \\
i
\end{array}\right) i ! S_{r, s}(p, i) \\
& =\prod_{j=1}^{p}\left(\begin{array}{c}
n+s-1+(j-1)(r-s) \\
s
\end{array}\right)-\prod_{j=1}^{p}\left(\begin{array}{c}
n+s-2+(j-1)(r-s) \\
s
\end{array}\right)
\end{aligned}
$$

and then the falling diagonal beginning in row $k=p r+1$ and column $j=s$, that is, the sequence $\prod_{j=1}^{p}\left(\begin{array}{c}n+s-1+(j-1)(r-s) \\ s\end{array}\right)$, can also be obtained as the partial sums of the falling 
diagonal beginning in the previous row (the one beginning in row $k=p r$ and column $j=s)$.

The case $r=s$ of (49) give us the "hyper-sums of powers of binomial coefficients", namely

$$
\sum_{i_{l}=1}^{n} \cdots \sum_{i_{2}=1}^{i_{3}} \sum_{i_{1}=1}^{i_{2}}\left(\begin{array}{c}
i_{1}+s-1 \\
s
\end{array}\right)^{p}=\frac{1}{(s !)^{p}} \sum_{i=s}^{p s}\left(\begin{array}{c}
n+s+l-1 \\
i+l
\end{array}\right) i ! S_{s, s}(p, i)
$$

Some examples from (49) are given in the following table:

\begin{tabular}{|c|c|c|}
\hline$(r, s, p)$ & $l$ & Sequence $(49)$ \\
\hline \hline$(3,2,2)$ & 1 & $\sum_{i=1}^{n}\left(\begin{array}{c}i+1 \\
2\end{array}\right)\left(\begin{array}{c}i+2 \\
2\end{array}\right)=3\left(\begin{array}{c}n+2 \\
3\end{array}\right)+9\left(\begin{array}{c}n+2 \\
4\end{array}\right)+6\left(\begin{array}{c}n+2 \\
5\end{array}\right)$. \\
\hline$(4,3,2)$ & 2 & $\sum_{j=1}^{n} \sum_{i=1}^{j}\left(\begin{array}{c}i+2 \\
3\end{array}\right)\left(\begin{array}{c}i+3 \\
3\end{array}\right)=4\left(\begin{array}{c}n+4 \\
5\end{array}\right)+24\left(\begin{array}{c}n+4 \\
6\end{array}\right)+40\left(\begin{array}{c}n+4 \\
7\end{array}\right)+20\left(\begin{array}{c}n+4 \\
8\end{array}\right)$. \\
\hline$(2,2,2)$ & 1 & $\sum_{i=0}^{n}\left(\begin{array}{c}i+1 \\
2\end{array}\right)=\left(\begin{array}{c}n+2 \\
3\end{array}\right)+6\left(\begin{array}{c}n+2 \\
4\end{array}\right)+6\left(\begin{array}{c}n+2 \\
5\end{array}\right)$. \\
\hline$(3,3,2)$ & 2 & $\sum_{j=1}^{n} \sum_{i=1}^{j}\left(\begin{array}{c}i+2 \\
3\end{array}\right)^{2}=\left(\begin{array}{c}n+4 \\
5\end{array}\right)+12\left(\begin{array}{c}n+4 \\
6\end{array}\right)+30\left(\begin{array}{c}n+4 \\
7\end{array}\right)+20\left(\begin{array}{c}n+4 \\
8\end{array}\right)$. \\
\hline
\end{tabular}

Table 3: Hockey-stick property on falling diagonals of a (r,s,p)-array.

Now let us consider the falling diagonals beginning in row $k=p r-s+1$ and column $j$, where $s<j \leqslant p s$. According to (29) these are the sequences (where $n \in \mathbb{N}$ )

$$
\frac{1}{(s !)^{p}} \sum_{i=s}^{p s}\left(\begin{array}{c}
n-1 \\
i-j
\end{array}\right) i ! S_{r, s}(p, i) \text {. }
$$

From (51) we see at once that the falling diagonal beginning in row $k=p r-s+1$ and column $j=s p$ is the constant sequence $\frac{(p s) !}{(s !)^{p}}$.

It turns out that the considered falling diagonals can be described in a different way.

Proposition 4. For $l=s, s+1, \ldots, s p$, and $n \in \mathbb{N}$ we have the identity

$$
\frac{1}{(s !)^{p}} \sum_{i=s}^{p s}\left(\begin{array}{c}
n-1 \\
i-l
\end{array}\right) i ! S_{r, s}(p, i)=\sum_{i=0}^{l}(-1)^{i}\left(\begin{array}{l}
l \\
i
\end{array}\right) \prod_{j=1}^{p}\left(\begin{array}{c}
n+l-1-i+(j-1)(r-s) \\
s
\end{array}\right) .
$$

Proof. By induction on $n$. For $n=1$ we have to prove that (for $s \leqslant l \leqslant s p$ )

$$
\sum_{i=0}^{l}(-1)^{i}\left(\begin{array}{l}
l \\
i
\end{array}\right) \prod_{j=1}^{p}\left(\begin{array}{c}
l-i+(j-1)(r-s) \\
s
\end{array}\right)=\frac{l !}{(s !)^{p}} S_{r, s}(p, l) .
$$


But this is an easy exercise left to the reader (by using the explicit formula (3) for $\left.S_{r, s}(p, l)\right)$. If $(52)$ is true for all positive integers $\leqslant n$, where $n \in \mathbb{N}$ is given, then

$$
\begin{aligned}
& \frac{1}{(s !)^{p}} \sum_{i=s}^{p s}\left(\begin{array}{c}
n \\
i-l
\end{array}\right) i ! S_{r, s}(p, i) \\
= & \frac{1}{(s !)^{p}} \sum_{i=s}^{p s}\left(\begin{array}{c}
n-1 \\
i-l
\end{array}\right) i ! S_{r, s}(p, i)+\frac{1}{(s !)^{p}} \sum_{i=s}^{p s}\left(\begin{array}{c}
n-1 \\
i-l-1
\end{array}\right) i ! S_{r, s}(p, i) \\
= & \sum_{i=0}^{l}(-1)^{i}\left(\begin{array}{c}
l \\
i
\end{array}\right) \prod_{j=1}^{p}\left(\begin{array}{c}
n+l-1-i+(j-1)(r-s) \\
s
\end{array}\right) \\
& +\sum_{i=0}^{l+1}(-1)^{i}\left(\begin{array}{c}
l+1 \\
i
\end{array}\right) \prod_{j=1}^{p}\left(\begin{array}{c}
n+l-i+(j-1)(r-s) \\
j
\end{array}\right) \\
= & \sum_{i=0}^{l+1}(-1)^{i}\left(\left(\begin{array}{c}
l+1 \\
i
\end{array}\right)-\left(\begin{array}{c}
l \\
i-1
\end{array}\right)\right) \prod_{j=1}^{p}\left(\begin{array}{c}
n+l-i+(j-1)(r-s) \\
l
\end{array}\right) \\
= & \sum_{i=0}^{l}(-1)^{i}\left(\begin{array}{l}
l \\
i
\end{array}\right) \prod_{j=1}^{p}\left(\begin{array}{c}
n+l-i+(j-1)(r-s) \\
s
\end{array}\right),
\end{aligned}
$$

as wanted.

The case $p=1$ of (52) says that for any $n, s \in \mathbb{N}$ we have

$$
\sum_{i=0}^{s}(-1)^{i}\left(\begin{array}{l}
s \\
i
\end{array}\right)\left(\begin{array}{c}
n+s-1-i \\
s
\end{array}\right)=1
$$

Also, if we set $l=s p$ in (52) (and use that the corresponding falling diagonal is the constant sequence $\left.\frac{(s p) !}{(s !)^{p}}\right)$, we obtain the following identity valid for any $n \in \mathbb{N}$

$$
\sum_{i=0}^{s p}(-1)^{i}\left(\begin{array}{c}
s p \\
i
\end{array}\right) \prod_{j=1}^{p}\left(\begin{array}{c}
n+s p-1-i+(j-1)(r-s) \\
s
\end{array}\right)=\frac{(s p) !}{(s !)^{p}}
$$

The case $r=s$ of (54) is

$$
\sum_{i=0}^{s p}(-1)^{i}\left(\begin{array}{c}
s p \\
i
\end{array}\right)\left(\begin{array}{c}
n-1+i \\
s
\end{array}\right)^{p}=(-1)^{s p} \frac{(s p) !}{(s !)^{p}}
$$

One more particular case of (52) is when $r=2 s$. By using (8) we can write (52) as

$$
\sum_{i=s}^{l+n-1}\left(\begin{array}{c}
s(p-1) \\
i-s
\end{array}\right)\left(\begin{array}{c}
n-1 \\
i-l
\end{array}\right)=\sum_{i=0}^{l}(-1)^{i}\left(\begin{array}{l}
l \\
i
\end{array}\right)\left(\begin{array}{c}
n+l-1-i+s(p-1) \\
s p
\end{array}\right) .
$$




\section{Rising diagonals}

In this section, related to rising diagonals of the $(r, s, p)$-arrays, Fibonacci and Lucas numbers will appear in a natural way. Recall that the Fibonacci numbers sequence $F_{n}$ and Lucas numbers sequence $L_{n}$ satisfy the same second-order recurrence $a_{n+2}=a_{n+1}+a_{n}$, with initial conditions $F_{0}=0$ and $F_{1}=1$ in the Fibonacci case, and $L_{0}=2$ and $L_{1}=1$ in the Lucas case. We will use without further comments the Binet's formulas for $F_{n}$ and $L_{n}$, namely $F_{n}=\frac{1}{\sqrt{5}}\left(\alpha^{n}-\beta^{n}\right)$ and $L_{n}=\alpha^{n}+\beta^{n}$, where $\alpha=\frac{1}{2}(1+\sqrt{5})$ and $\beta=\frac{1}{2}(1-\sqrt{5})$ (observe that $\alpha+\beta=1$; we will use this in the proof of lemma 6 ).

We consider the polynomials

$$
\sum_{k=r}^{r p} k ! S_{r, r}(p, k) x^{k-r}
$$

Proposition 5. The following formula holds

$$
\sum_{k=r}^{r p} k ! S_{r, r}(p, k) x^{k-r}=\sum_{k=r}^{r p}(-1)^{k-r p} k ! S_{r, r}(p, k)(x+1)^{k-r} .
$$

Proof. By induction on $p$. For $p=1$ it is easy to see that both sides of (58) are equal to $r$ !. Let us assume that (58) is true for a given $p \in \mathbb{N}$. Then, by using (7) we have that

$$
\begin{aligned}
\sum_{k=r}^{r(p+1)} k ! S_{r, r}(p+1, k) x^{k-r} & =\sum_{k=r}^{r(p+1)} k ! \sum_{i=0}^{r}\left(\begin{array}{c}
k+i-r \\
i
\end{array}\right) r^{i} S_{r, r}(p, k+i-r) x^{k-r} \\
& =\sum_{i=0}^{r}\left(\begin{array}{l}
r \\
i
\end{array}\right) \sum_{k=r}^{r p} \frac{(k+r-i) !}{(k-i) !} k ! S_{r, r}(p, k) x^{k-i} \\
& =\sum_{i=0}^{r}\left(\begin{array}{l}
r \\
i
\end{array}\right) \sum_{k=r}^{r p}\left(\frac{d^{r}}{d x^{r}} x^{k+r-i}\right) k ! S_{r, r}(p, k) \\
& =\frac{d^{r}}{d x^{r}}\left(x^{2 r} \sum_{i=0}^{r}\left(\begin{array}{c}
r \\
i
\end{array}\right) x^{-i} \sum_{k=r}^{r p} k ! S_{r, r}(p, k) x^{k-r}\right) \\
& =\frac{d^{r}}{d x^{r}}\left(x^{r}(x+1)^{r} \sum_{k=r}^{r p} k ! S_{r, r}(p, k) x^{k-r}\right) \\
& =\frac{d^{r}}{d x^{r}}\left(x^{r}(x+1)^{r} \sum_{k=r}^{r p}(-1)^{k-r p} k ! S_{r, r}(p, k)(x+1)^{k-r}\right) .
\end{aligned}
$$

Some further simplifications give us

$$
\sum_{k=r}^{r(p+1)} k ! S_{r, r}(p+1, k) x^{k-r}
$$




$$
\begin{aligned}
= & \frac{d^{r}}{d x^{r}}\left(x^{r} \sum_{k=r}^{r p}(-1)^{k-r p} k ! S_{r, r}(p, k)(x+1)^{k}\right) \\
= & \frac{d^{r}}{d x^{r}}\left(\sum_{i=0}^{r}\left(\begin{array}{l}
r \\
i
\end{array}\right)(-1)^{i}(x+1)^{r-i} \sum_{k=r}^{r p}(-1)^{k-r p} k ! S_{r, r}(p, k)(x+1)^{k}\right) \\
= & \sum_{i=0}^{r}\left(\begin{array}{l}
r \\
i
\end{array}\right) \sum_{k=r}^{r p}(-1)^{k-i-r p} k ! S_{r, r}(p, k) \frac{d^{r}}{d x^{r}}(x+1)^{k+r-i} \\
= & \sum_{i=0}^{r}\left(\begin{array}{l}
r \\
i
\end{array}\right) \sum_{k=r}^{r p}(-1)^{k+r-i-r(p+1)}(k+r-i) ! \frac{k !}{(k-i) !} S_{r, r}(p, k)(x+1)^{k-i} \\
= & \sum_{k=r}^{r(p+1)}(-1)^{k-r(p+1)} k ! \sum_{i=0}^{r}\left(\begin{array}{c}
k+i-r \\
i
\end{array}\right) \frac{r !}{(r-i) !} S_{r, r}(p, k+i-r)(x+1)^{k-r} \\
= & \sum_{k=r}^{r(p+1)}(-1)^{k-r(p+1)} k ! S_{r, r}(p+1, k)(x+1)^{k-r},
\end{aligned}
$$

as wanted (we used (7) in the last step) .

Lemma 6. (a) If $r$ is even, or $r$ and $p$ are odd, we have

$$
\sum_{k=r}^{r p}(-1)^{k} k ! S_{r, r}(p, k) F_{k-r}=0 .
$$

(b) If $p$ is even and $r$ is odd, we have

$$
\sum_{k=r}^{r p}(-1)^{k} k ! S_{r, r}(p, k) L_{k-r}=0
$$

Proof. In (58) set $x=-\alpha$ to obtain

$$
\sum_{k=r}^{r p}(-1)^{k-r} k ! S_{r, r}(p, k) \alpha^{k-r}=\sum_{k=r}^{r p}(-1)^{k-r p} k ! S_{r, r}(p, k) \beta^{k-r} .
$$

The desired formulas (59) and (60) are obtained from (61) and the hypotheses made on $r$ and $p$ in each case.

Lemma 7. (a) The following identity holds

$$
\sum_{j=0}^{r(p-1)} \sum_{i=r}^{r+j}\left(\begin{array}{c}
r(p-1)-j-1 \\
r+j-i
\end{array}\right) i ! S_{r, r}(p, i)=\sum_{i=r}^{r p-1} i ! S_{r, r}(p, i) F_{r p-i} .
$$

(b) Let $r, l \in \mathbb{N}$ be given, such that $l \geqslant r$. Then

$$
\sum_{i=0}^{l}(-1)^{i+1}\left(\begin{array}{c}
r \\
l-i
\end{array}\right) F_{i}=F_{2 r-l}
$$


Proof. (a) Some simple manipulations on the left hand side of (62) give us

$$
\begin{aligned}
& \sum_{j=0}^{r(p-1)-1} \sum_{i=r}^{r+j}\left(\begin{array}{c}
r(p-1)-j-1 \\
r+j-i
\end{array}\right) i ! S_{r, r}(p, i) \\
= & \sum_{j=0}^{r(p-1)-1} \sum_{i=0}^{r(p-1)-1}\left(\begin{array}{c}
r(p-1)-j-1 \\
j-i
\end{array}\right)(i+r) ! S_{r, r}(p, i+r) \\
= & \sum_{i=0}^{r(p-1)-1}(i+r) ! S_{r, r}(p, i+r) \sum_{j=0}^{r(p-1)-1-i}\left(\begin{array}{c}
r(p-1)-j-i-1 \\
j
\end{array}\right) \\
= & \sum_{i=r}^{r p-1} i ! S_{r, r}(p, i) F_{r p-i},
\end{aligned}
$$

as wanted.

(b) We begin with the left-hand side of (63) and Binet's formulas to write

$$
\begin{aligned}
\sum_{i=0}^{l}(-1)^{i+1}\left(\begin{array}{c}
r \\
l-i
\end{array}\right) F_{i} & =\frac{(-1)^{l+1}}{\sqrt{5}} \sum_{i=0}^{r}(-1)^{i}\left(\begin{array}{c}
r \\
i
\end{array}\right)\left(\alpha^{l-i}-\beta^{l-i}\right) \\
& =\frac{(-1)^{l+1}}{\sqrt{5}}\left(\alpha^{l-r} \sum_{i=0}^{r}(-1)^{i}\left(\begin{array}{c}
r \\
i
\end{array}\right) \alpha^{r-i}-\beta^{l-r} \sum_{i=0}^{r}(-1)^{i}\left(\begin{array}{l}
r \\
i
\end{array}\right) \beta^{r-i}\right) \\
& =\frac{(-1)^{l+1}}{\sqrt{5}}\left(\alpha^{l-2 r}-\beta^{l-2 r}\right) \\
& =F_{2 r-l},
\end{aligned}
$$

as wanted.

In the following proposition we will use the identities

$$
\begin{aligned}
& F_{r(p-1)} F_{2 r-l}=F_{r} F_{r p-l}+(-1)^{r} F_{r(p-2)} F_{r-l}, \\
& L_{r(p-1)} F_{2 r-l}=L_{r} F_{r p-l}-(-1)^{r} L_{r-l} F_{r(p-2)},
\end{aligned}
$$

which are consequences of the index-reduction formula

$$
G_{M} F_{N}-G_{M+K} F_{N-K}=(-1)^{N-K} G_{M+K-N} F_{K},
$$

where $G=F$ or $G=L$.

Proposition 8. For $k \geqslant 2 r(p-1)$ we have

$$
\sum_{j=0}^{\left\lfloor\frac{k-1}{2}\right\rfloor} \sum_{i=r}^{r+j}\left(\begin{array}{c}
k-j-r(p-1)-1 \\
r+j-i
\end{array}\right) i ! S_{r, r}(p, i)
$$




$$
= \begin{cases}\left(\frac{1}{F_{r}} \sum_{i=r}^{r(p+1)}(-1)^{i+r+1}(i-r) ! S_{r, r}(p+1, i) F_{i-r}\right) F_{k-r(p-1)} & \begin{array}{l}
r \text { is even } \\
\text { or } \\
r \text { and } p \text { are odd }
\end{array} \\
\left(\frac{1}{L_{r}} \sum_{i=r}^{r(p+1)}(-1)^{i}(i-r) ! S_{r, r}(p+1, i) F_{i-r}\right) L_{k-r(p-1)} & \begin{array}{l}
r \text { is odd } \\
\text { and } \\
p \text { is even }
\end{array}\end{cases}
$$

Proof. Let us prove first that if $r$ is even or $r$ and $p$ are odd the formula

$$
\begin{aligned}
& \sum_{j=0}^{\left\lfloor\frac{k-1}{2}\right\rfloor} \sum_{i=r}^{r+j}\left(\begin{array}{c}
k-j-r(p-1)-1 \\
r+j-i
\end{array}\right) i ! S_{r, r}(p, i) \\
& =\left(\frac{1}{F_{r}} \sum_{i=r}^{r(p+1)}(-1)^{i+r+1}(i-r) ! S_{r, r}(p+1, i) F_{i-r}\right) F_{k-r(p-1)},
\end{aligned}
$$

holds for $k \geqslant 2 r(p-1)$. We proceed by induction on $k$. For $k=2 r(p-1)$ we have to prove that

$$
\begin{aligned}
& \sum_{j=0}^{r(p-1)} \sum_{i=r}^{r+j}\left(\begin{array}{c}
r(p-1)-j-1 \\
r+j-i
\end{array}\right) i ! S_{r, r}(p, i) \\
& =\left(\frac{1}{F_{r}} \sum_{i=r}^{r(p+1)}(-1)^{i+r+1}(i-r) ! S_{r, r}(p+1, i) F_{i-r}\right) F_{r(p-1)} .
\end{aligned}
$$

That is, according to (62) we have to prove that

$$
\left(\frac{1}{F_{r}} \sum_{i=r}^{r(p+1)}(-1)^{i+r+1}(i-r) ! S_{r, r}(p+1, i) F_{i-r}\right) F_{r(p-1)}=\sum_{i=r}^{r p-1} i ! S_{r, r}(p, i) F_{r p-i}
$$

We begin with the left-hand side of (69) to write

$$
\begin{aligned}
& \left(\frac{1}{F_{r}} \sum_{i=r}^{r(p+1)}(-1)^{i+r+1}(i-r) ! S_{r, r}(p+1, i) F_{i-r}\right) F_{r(p-1)} \\
& =\frac{F_{r(p-1)}}{F_{r}} \sum_{i=r}^{r(p+1)}(-1)^{i+r+1}(i-r) ! \sum_{j=0}^{r}\left(\begin{array}{c}
i+j-r \\
j
\end{array}\right) \frac{r !}{(r-j) !} S_{r, r}(p, i+j-r) F_{i-r} \\
& =\frac{F_{r(p-1)}}{F_{r}} \sum_{i=0}^{r p}(-1)^{i+1} i ! \sum_{j=0}^{r}\left(\begin{array}{c}
i+j \\
j
\end{array}\right) \frac{r !}{(r-j) !} S_{r, r}(p, i+j) F_{i} \\
& =\frac{F_{r(p-1)}}{F_{r}} \sum_{i=0}^{r p}(-1)^{i+1} \sum_{j=0}^{r}\left(\begin{array}{l}
r \\
j
\end{array}\right)(i+j) ! S_{r, r}(p, i+j) F_{i}
\end{aligned}
$$




$$
\begin{aligned}
& =\frac{F_{r(p-1)}}{F_{r}} \sum_{i=0}^{r p}(-1)^{i+1} \sum_{l=0}^{r p}\left(\begin{array}{c}
r \\
l-i
\end{array}\right) l ! S_{r, r}(p, l) F_{i} \\
& =\frac{F_{r(p-1)}}{F_{r}} \sum_{l=0}^{r p} l ! S_{r, r}(p, l) F_{2 r-l}
\end{aligned}
$$

where we used (63) in the last step. Now use (64) and (59) to obtain finally that if $r$ is even or $r$ and $p$ are odd we have

$$
\begin{aligned}
& \left(\frac{1}{F_{r}} \sum_{i=r}^{r(p+1)}(-1)^{i+r+1}(i-r) ! S_{r, r}(p+1, i) F_{i-r}\right) F_{r(p-1)} \\
= & \frac{1}{F_{r}} \sum_{l=0}^{r p} l ! S_{r, r}(p, l)\left(F_{r} F_{r p-l}+(-1)^{r} F_{r(p-2)} F_{r-l}\right) \\
= & \sum_{l=0}^{r p} l ! S_{r, r}(p, l) F_{r p-l},
\end{aligned}
$$

as expected. The rest of the induction argument is an easy exercise (left to the reader) by using the recurrence of Fibonacci numbers (see Proposition 13 in [24]).

Now we prove that if $r$ is odd and $p$ is even we have

$$
\begin{aligned}
& \sum_{j=0}^{\left\lfloor\frac{k-1}{2}\right\rfloor} \sum_{i=r}^{r+j}\left(\begin{array}{c}
k-j-r(p-1)-1 \\
r+j-i
\end{array}\right) i ! S_{r, r}(p, i) \\
& =\left(\frac{1}{L_{r}} \sum_{i=r}^{r(p+1)}(-1)^{i}(i-r) ! S_{r, r}(p+1, i) F_{i-r}\right) L_{k-r(p-1)} \text {. }
\end{aligned}
$$

For $k=2 r(p-1)$ we have to prove (according to (62)) that if $r$ is odd and $p$ is even:

$$
\left(\frac{1}{L_{r}} \sum_{i=r}^{r(p+1)}(-1)^{i}(i-r) ! S_{r, r}(p+1, i) F_{i-r}\right) L_{r(p-1)}=\sum_{i=r}^{r p-1} i ! S_{r, r}(p, i) F_{r p-i} .
$$

We have

$$
\begin{aligned}
& \left(\frac{1}{L_{r}} \sum_{i=r}^{r(p+1)}(-1)^{i}(i-r) ! S_{r, r}(p+1, i) F_{i-r}\right) L_{r(p-1)} \\
= & \frac{L_{r(p-1)}}{L_{r}} \sum_{i=0}^{r p}(-1)^{i+r} i ! \sum_{j=0}^{r}\left(\begin{array}{c}
i+j \\
j
\end{array}\right) \frac{r !}{(r-j) !} S_{r, r}(p, i+j) F_{i} \\
= & \frac{L_{r(p-1)}}{L_{r}} \sum_{i=0}^{r p}(-1)^{i+r} \sum_{j=0}^{r}\left(\begin{array}{l}
r \\
j
\end{array}\right)(i+j) ! S_{r, r}(p, i+j) F_{i}
\end{aligned}
$$




$$
\begin{aligned}
& =\frac{L_{r(p-1)}}{L_{r}} \sum_{i=0}^{r p}(-1)^{i+r} \sum_{l=r}^{r p}\left(\begin{array}{c}
r \\
l-i
\end{array}\right) l ! S_{r, r}(p, l) F_{i} \\
& =\frac{L_{r(p-1)}}{L_{r}} \sum_{l=r}^{r p} l ! S_{r, r}(p, l) F_{2 r-l},
\end{aligned}
$$

where we used that $r$ is odd and (63). Now use (65) and (60) to conclude finally that for $r$ odd and $p$ even we have

$$
\begin{aligned}
& \left(\frac{1}{L_{r}} \sum_{i=r}^{r(p+1)}(-1)^{i}(i-r) ! S_{r, r}(p+1, i) F_{i-r}\right) L_{r(p-1)} \\
= & \frac{1}{L_{r}} \sum_{l=r}^{r p} l ! S_{r, r}(p, l)\left(L_{r} F_{r p-l}+L_{r-l} F_{r(p-2)}\right) \\
= & \sum_{l=r}^{r p} l ! S_{r, r}(p, l) F_{r p-l}
\end{aligned}
$$

as wanted. Again, the rest of the induction argument is an easy exercise left to the reader.

According to (29), the sum of the elements of the rising diagonal beginning in row $k \geqslant 2 r(p-1)$ and column $j=s$ is

$$
\frac{1}{(r !)^{p}} \sum_{j=0}^{\left\lfloor\frac{k-1}{2}\right\rfloor} \sum_{i=r}^{r+j}\left(\begin{array}{c}
k-j-r(p-1)-1 \\
r+j-i
\end{array}\right) i ! S_{r, r}(p, i)
$$

Thus, in proposition 8 we have proved that when $r$ is even or $r$ and $p$ are odd, the sum $(72)$ is equal to

$$
\left(\frac{1}{(r !)^{p} F_{r}} \sum_{i=r}^{r(p+1)}(-1)^{i+r+1}(i-r) ! S_{r, r}(p+1, i) F_{i-r}\right) F_{k-r(p-1)},
$$

and that when $r$ is odd and $p$ is even, this sum is equal to

$$
\left(\frac{1}{(r !)^{p} L_{r}} \sum_{i=r}^{r(p+1)}(-1)^{i}(i-r) ! S_{r, r}(p+1, i) F_{i-r}\right) L_{k-r(p-1)} .
$$

When $r=1$ the expressions in parenthesis in (73) and (74) are equal to the $p$-sequence

$$
\left(\sum_{i=1}^{p+1}(-1)^{i}(i-1) ! S(p+1, i) F_{i-1}\right)_{p \in \mathbb{N}}=(1,1,7,13,151,421, \ldots) \text {, }
$$


called "Stirling-Bernoulli transform of Fibonacci numbers" ([25, A050946]). This is the sequence that appears in [24] and the result is that (for $k \geqslant 2(p-1)$ ) the sum of rising diagonals is equal to the Stirling-Bernoulli transform (75) times the Fibonacci number $F_{k-p+1}$ when $p$ is odd, and times the Lucas number $L_{k-p+1}$ when $p$ is even. For $r>1$ we have the following generalizations of the sequence (75), which we call " $r$-Stirling-Bernoulli transform of Fibonacci numbers":

1. If $r$ is even, the $r$-Stirling-Bernoulli transform of Fibonacci numbers is the $p$-sequence

$$
\left(\frac{1}{(r !)^{p} F_{r}} \sum_{i=r}^{r(p+1)}(-1)^{i+r+1}(i-r) ! S_{r, r}(p+1, i) F_{i-r}\right)_{p \in \mathbb{N}} .
$$

For example, if $r=2$ the sequence is $(1,7,115,3499,170611, \ldots)$ and if $r=4$ the sequence is $(1,91,54091,116359591, \ldots)$. In this case the result on rising diagonals is the following:

In a $(r, r, p)$-array, where $r$ is even, the sum of the elements of the rising diagonals (beginning in row $k \geqslant 2 r(p-1)$ and column $r$ ) is equal to the $r$-Stirling-Bernoulli transform of Fibonacci numbers (76) times the Fibonacci number $F_{k-r(p-1)}$.

2. If $r$ is odd, we have two versions of $r$-Stirling-Bernoulli transforms of Fibonacci numbers, depending on the parity of $p$ :

(a) For $p$ odd the sequence is

$$
\left(\frac{1}{(r !)^{p} F_{r}} \sum_{i=r}^{r(p+1)}(-1)^{i+r+1}(i-r) ! S_{r, r}(p+1, i) F_{i-r}\right)_{p=1,3,5, \ldots}
$$

and the result is the following:

In a $(r, r, p)$-array, where $r$ and $p$ are odd, the sum of the elements of the rising diagonals (beginning in row $k \geqslant 2 r(p-1)$ and column $r$ ) is equal to the $r$-Stirling-Bernoulli transform of Fibonacci numbers (7r) times the Fibonacci number $F_{k-r(p-1)}$.

(b) For $p$ even the sequence is

$$
\left(\frac{1}{(r !)^{p} L_{r}} \sum_{i=r}^{r(p+1)}(-1)^{i+r+1}(i-r) ! S_{r, r}(p+1, i) F_{i-r}\right)_{p=2,4,6, \ldots}
$$

and the result is the following:

In a $(r, r, p)$-array, where $r$ is odd and $p$ is even, the sum of the elements of the rising diagonals (beginning in row $k \geqslant 2 r(p-1)$ and column $r$ ) is equal to the r-Stirling-Bernoulli transform of Fibonacci numbers (78) times the Lucas number $L_{k-r(p-1)}$. 
Note that in the case $r=1$, the two versions of $r$-Stirling-Bernoulli transforms of Fibonacci numbers (77) and (78) are the odd-indexed and even-indexed subsequences of the sequence (75), respectively. (This is the case of [24].) However, if $r>1$ these sequences are different. For example, if $r=3$ the corresponding sequences (77) and (78) are $(1,2371,303607891, \ldots)$ and $(11,264431,124768775351, \ldots)$, respectively. Thus, in the $(3,3, p)$-array, the sum of the elements of the rising diagonals (beginning in row $k \geqslant 6(p-1)$ and 3rd column) is equal to $F_{k}$ when $p=1$, to $11 L_{k-3}$ when $p=2$, to $2371 F_{k-6}$ when $p=3$, to $264431 L_{k-9}$ when $p=4$, to $303607891 F_{k-12}$ when $p=5$, and so on.

In tables 4 and 5 we have some more examples.

\begin{tabular}{|c|c|c|c|c|c|}
\hline$r$ & $p$ & $\begin{array}{r}\text { Beginning } \\
\text { in row } k\end{array}$ & Rising diagonal & $\begin{array}{c}\text { Sum } \\
\text { of } \\
\text { elements }\end{array}$ & Equal to \\
\hline \multirow[t]{4}{*}{2} & 2 & $k=4$ & $(1,6)$ & 7 & $7 F_{2}$ \\
\hline & & $k=5$ & $(1,7,6)$ & 14 & $7 F_{3}$ \\
\hline & & $k=6$ & $(1,8,12)$ & 21 & $7 F_{4}$ \\
\hline & & $\vdots$ & $\vdots$ & $\vdots$ & $\vdots$ \\
\hline \multirow[t]{4}{*}{2} & 3 & $k=8$ & $(1,26,138,180)$ & 345 & $115 F_{4}$ \\
\hline & & $k=9$ & $(1,27,163,294,90)$ & 575 & $115 F_{5}$ \\
\hline & & $k=10$ & $(1,28,189,432,270)$ & 920 & $115 F_{6}$ \\
\hline & & $\vdots$ & $\vdots$ & $\vdots$ & \\
\hline \multirow[t]{4}{*}{3} & 3 & $k=12$ & $(1,64,873,4380,8610,5040)$ & 18968 & $2371 F_{6}$ \\
\hline & & $k=13$ & $(1,65,936,5191,12240,10710,1680)$ & 30823 & $2371 F_{7}$ \\
\hline & & $k=14$ & $(1,66,1000,6064,16620,19320,6720)$ & 49791 & $2371 F_{8}$ \\
\hline & & $\vdots$ & $\vdots$ & $\vdots$ & \\
\hline \multirow[t]{4}{*}{4} & 2 & $k=8$ & $(1,22,110,140)$ & 273 & $91 F_{4}$ \\
\hline & & $k=9$ & $(1,23,131,230,70)$ & 455 & $91 F_{5}$ \\
\hline & & $k=10$ & $(1,24,153,340,210)$ & 728 & $91 F_{6}$ \\
\hline & & $\vdots$ & $\vdots$ & $\vdots$ & \\
\hline
\end{tabular}

Table 4: Sums of rising diagonals in a (r,r,p)-array, when $\mathrm{r}$ is even, or $\mathrm{r}$ and $\mathrm{p}$ are odd.

Remark 9. We believe that for the general case $r>s$, the sums of rising diagonals in a $(r, s, p)$-arrays are also related with Fibonacci and/or Lucas numbers. Note that the sum of the elements of the rising diagonal beginning in column $s$ and row $k \geqslant(s+r) p-2 s$ is given by

$$
\frac{1}{(s !)^{p}} \sum_{j=0}^{\left\lfloor\frac{k-1-(r-s) p}{2}\right\rfloor} \sum_{i=s}^{s+j}\left(\begin{array}{c}
k-j-p r+s-1 \\
s+j-i
\end{array}\right) i ! S_{r, s}(p, i) .
$$

In the case $r=2 s$ it is not difficult to see that the sum $(79)$ is equal to $\frac{(s p) !}{(s !)^{p}} F_{k-s p}$ (since, 


\begin{tabular}{|c|c|c|c|c|c|}
\hline$r$ & $p$ & $\begin{array}{c}\text { Beginning } \\
\text { in row k }\end{array}$ & The rising diagonal is & $\begin{array}{c}\text { Sum } \\
\text { of } \\
\text { elements }\end{array}$ & Equal to \\
\hline \hline 3 & 2 & $k=6$ & $(1,13,30)$ & 44 & $11 L_{3}$ \\
\hline & & $k=7$ & $(1,14,42,20)$ & 77 & $11 L_{4}$ \\
\hline & & $k=8$ & $(1,15,55,50)$ & 121 & $11 L_{5}$ \\
\hline & & $\vdots$ & $\vdots$ & $\vdots$ & $\vdots$ \\
\hline 3 & 4 & $k=18$ & $(1,259,10257,150000, \ldots, 7203420,6186600,1663200)$ & 20096756 & $264431 L_{9}$ \\
\hline & & $k=19$ & $(1,260,10515,160000, \ldots, 11040960,4762800,369600)$ & 32525013 & $264431 L_{10}$ \\
\hline & & $k=20$ & $(1,261,10774,170257, \ldots, 18244380,10949400,2032800)$ & 52621769 & $264431 L_{11}$ \\
\hline & & $\vdots$ & $\vdots$ & $\vdots$ & \\
\hline 5 & 2 & $k=10$ & $(1,33,271,770,630)$ & 1705 & $155 L_{5}$ \\
\hline & & $k=11$ & $(1,34,303,1010,1190,252)$ & 2790 & $155 L_{6}$ \\
\hline & & $k=12$ & $(1,35,336,1281,1960,882)$ & 4495 & $155 L_{7}$ \\
\hline & & $\vdots$ & $\vdots$ & $\vdots$ & \\
\hline
\end{tabular}

Table 5: Sums of rising diagonals in a (r,r,p)-array, when $\mathrm{r}$ is odd and $\mathrm{p}$ is even.

as we noticed in section 2, the $(2 s, s, p)$-array is a piece of Pascal's triangle multiplied by $\frac{(s p) !}{(s !)^{p}}$.) Then, we have for $k \geqslant 3 s p-2 s$ that

$$
\frac{1}{(s !)^{p}} \sum_{j=0}^{\left\lfloor\frac{k-1-s p}{2}\right\rfloor} \sum_{i=s}^{s+j}\left(\begin{array}{c}
k-j-2 s p+s-1 \\
s+j-i
\end{array}\right) i ! S_{2 s, s}(p, i)=\frac{(s p) !}{(s !)^{p}} F_{k-s p}
$$

However, formula (80) is not an interesting result, since we can write it (by using (8)) as

$$
\sum_{j=0}^{\left\lfloor\frac{k-1-s p}{2}\right\rfloor} \sum_{i=s}^{s+j}\left(\begin{array}{c}
k-j-2 s p+s-1 \\
s+j-i
\end{array}\right)\left(\begin{array}{c}
s(p-1) \\
i-s
\end{array}\right)=F_{k-s p}
$$

and one can see at once that (81) is consequence of the following two easy to prove identities

$$
\sum_{j=0}^{m}\left(\begin{array}{c}
m-j \\
j
\end{array}\right)=F_{m+1}, \quad \text { and } \quad \sum_{i=0}^{m}\left(\begin{array}{c}
m \\
i
\end{array}\right) F_{r-i}=F_{m+r},
$$

where $m$ is a non-negative integer and $r \in \mathbb{Z}$. We obtained explicit values of the sum (79) for some concrete values of $r, s, p, k$ in the remaining cases (when $1 \leqslant s<r, r \neq 2 s$ ) and observed that Fibonacci and/or Lucas numbers appear in these sums. In table 6 we have some examples.

We mention that we were not able to propose and prove the results (of the last column of Table 6) corresponding to this general situation. We believe that they are not as nice and simple as those we showed in this section for the case $r=s$. 


\begin{tabular}{|c|c|c|c|c|c|c|}
\hline$r$ & $s$ & $p$ & $\begin{array}{l}\text { Beginning in } \\
\text { column } j \\
\text { and row } k\end{array}$ & The rising diagonals are & $\begin{array}{l}\text { Sum of ele- } \\
\text { ments }\end{array}$ & Equals to \\
\hline 3 & 1 & 2 & $\begin{array}{l}k=6 \\
k=7 \\
k=8, \ldots\end{array}$ & $\begin{array}{l}(3), \\
(3,2), \\
(3,5), \ldots\end{array}$ & $\begin{array}{l}3, \\
5, \\
8, \ldots\end{array}$ & $\begin{array}{l}F_{4}, \\
F_{5}, \\
F_{6}, \ldots \\
\end{array}$ \\
\hline & & 3 & $\begin{array}{l}k=10 \\
k=11 \\
k=12, \ldots\end{array}$ & $\begin{array}{l}(15,18), \\
(15,33,6), \\
(15,48,24), \ldots\end{array}$ & $\begin{array}{l}33 \\
54, \\
87, \ldots\end{array}$ & $\begin{array}{l}3 L_{5} \\
3 L_{6} \\
3 L_{7}, \ldots\end{array}$ \\
\hline & 2 & 3 & $\begin{aligned} k & =11 \\
k & =12 \\
k & =13, \ldots\end{aligned}$ & $\begin{array}{l}(18,162,414,270), \\
(18,180,558,558,90), \\
(18,198,720,972,360), \ldots\end{array}$ & $\begin{array}{l}864, \\
1404, \\
2268, \ldots\end{array}$ & $\begin{array}{l}108 F_{6} \\
108 F_{7} \\
108 F_{8}, \ldots\end{array}$ \\
\hline 4 & 1 & 2 & $\begin{aligned} k & =8 \\
k & =9 \\
k & =10, \ldots\end{aligned}$ & $\begin{array}{l}(4) \\
(4,2) \\
(4,6), \ldots\end{array}$ & $\begin{array}{l}4, \\
6, \\
10, \ldots\end{array}$ & $\begin{array}{l}2 F_{3} \\
2 F_{4} \\
2 F_{5}, \ldots\end{array}$ \\
\hline & & 3 & $\begin{aligned} k & =13 \\
k & =14 \\
k & =15, \ldots\end{aligned}$ & $\begin{array}{l}(28,24), \\
(28,52,6), \\
(28,80,30), \ldots\end{array}$ & $\begin{array}{l}52 \\
86, \\
138, .\end{array}$ & $\begin{array}{l}2\left(7 F_{4}+F_{5}\right), \\
2\left(7 F_{5}+F_{6}\right), \\
2\left(7 F_{6}+F_{7}\right), \ldots\end{array}$ \\
\hline & 3 & 3 & $\begin{array}{l}k=15, \\
k=16, \\
k=17, .\end{array}$ & $\begin{array}{l}(40,800,5440,15840,19040,6720), \\
(40,840,6200,20560,30840,17360,1680), \\
(40,880,7000,26000,46680,36400,8400),\end{array}$ & $\begin{array}{l}47880, \\
77520 \\
125400\end{array}$ & $\begin{array}{l}2280 F_{8} \\
2280 F_{9} \\
2280 F_{10}, \ldots\end{array}$ \\
\hline
\end{tabular}

Table 6: Sums of rising diagonals in (r,s,p)-arrays.

\section{Some additional results}

By equating coefficients of similar powers of $x$ in both sides of (58) we see that

$$
S_{r, r}(p, j)=\frac{1}{j !} \sum_{i=j}^{r p}(-1)^{i-r p}\left(\begin{array}{c}
i-r \\
j-r
\end{array}\right) i ! S_{r, r}(p, i) .
$$

In this section we want to obtain some consequences of this identity. First of all, observe that (15) and (83) give us that

$$
\left(\begin{array}{l}
n \\
s
\end{array}\right)^{p}=\frac{1}{(s !)^{p}} \sum_{k=s}^{s p} \sum_{i=k}^{s p}(-1)^{i-s p} i ! S_{s, s}(p, i)\left(\begin{array}{l}
i-s \\
k-s
\end{array}\right)\left(\begin{array}{l}
n \\
k
\end{array}\right),
$$

which can be written as

$$
\left(\begin{array}{l}
n \\
s
\end{array}\right)^{p}=\frac{(-1)^{s p}}{(s !)^{p}} \sum_{k=s}^{s p}(-1)^{k} k ! S_{s, s}(p, k)\left(\begin{array}{c}
n+k-s \\
k
\end{array}\right) .
$$

After a simplification procedure (that we describe next), using Pascal's triangle recurrence, the right-hand side of (85) can be written in a simpler form, in which we need only 'the half' of the number of coefficients involved in (85). To prove this fact is one of the goals of this section, and it is contained in corollary 11.

The mentioned simplification procedure is as follows.

Procedure: Begin with expression (85) (this is our "starting point" expression) and perform steps $1,2,3$. 
Step 1 Write the last (the $k=s p$-th) term of the right-hand side of (85), namely the term $\frac{(s p) !}{(s !)^{p}} S_{s, s}(p, s p)\left(\begin{array}{c}n+s p-s \\ s p\end{array}\right)$, as

$$
\frac{(s p) !}{(s !)^{p}} S_{s, s}(p, s p)\left(\left(\begin{array}{c}
n+s p-s-1 \\
s p-1
\end{array}\right)+\left(\begin{array}{c}
n+s p-s-1 \\
s p
\end{array}\right)\right) .
$$

Step 2 Write together the first summand of (86), namely $\frac{(s p) !}{(s !)^{p}} S_{s, s}(p, s p)\left(\begin{array}{c}n+s p-s-1 \\ s p-1\end{array}\right)$, and the $k=(s p-1)$-th summand of $(85)$, namely $-\frac{(s p-1) !}{(s !)^{p}} S_{s, s}(p, s p-1)\left(\begin{array}{c}n+s p-s-1 \\ s p-1\end{array}\right)$, to get the new $k=(s p-1)$-th summand of $(85)$ as

$$
\left(\frac{(s p) !}{(s !)^{p}} S_{s, s}(p, s p)-\frac{(s p-1) !}{(s !)^{p}} S_{s, s}(p, s p-1)\right)\left(\begin{array}{c}
n+s p-s-1 \\
s p-1
\end{array}\right)
$$

Step 3 Return to Step 1 with the new $k=(s p-1)$-th summand (87), to get (after the new Step 2) the new $k=(s p-2)$-th summand as

$$
\begin{aligned}
& \left(\frac{(s p) !}{(s !)^{p}} S_{s, s}(p, s p)-\frac{(s p-1) !}{(s !)^{p}} S_{s, s}(p, s p-1)+\frac{(s p-2) !}{(s !)^{p}} S_{s, s}(p, s p-2)\right) \\
& \times\left(\begin{array}{c}
n+s p-s-2 \\
s p-2
\end{array}\right)
\end{aligned}
$$

and so on. That is, return to Step 1 with the new $(s p-2)$-th summand (88) to get a new $(s p-3)$-th summand. Continue in this way until you perform Step 2 involving the first term (the $k=s$-th term) of the right-hand side of (85).

At the end of Procedure we have the expression (85) transformed in

$$
\begin{aligned}
\left(\begin{array}{c}
n \\
s
\end{array}\right)^{p}= & \frac{(-1)^{s p}}{(s !)^{p}} \sum_{t=0}^{1} \sum_{j=s+t}^{s p}(-1)^{j} j ! S_{s, s}(p, j)\left(\begin{array}{c}
n \\
s+t
\end{array}\right) \\
& +\frac{(-1)^{s p}}{(s !)^{p}} \sum_{t=2}^{s(p-1)}\left(\sum_{j=s+t}^{s p}(-1)^{j} j ! S_{s, s}(p, j)\right)\left(\begin{array}{c}
n+t-1 \\
s+t
\end{array}\right) .
\end{aligned}
$$

Now we take (89) as the starting point and repeat Procedure, beginning with the last term of the right-hand side of (89) and going backwards, up to the third term (that is, we leave the first two summands of the right-hand side of (89) as they appear in this expression, namely $\frac{(-1)^{s p}}{(s !)^{p}} \sum_{t=0}^{1} \sum_{j=s+t}^{s p}(-1)^{j} j ! S_{s, s}(p, j)\left(\begin{array}{c}n \\ s+t\end{array}\right)$ ). (The reason why we stop now in the third term will become clear shortly, and will be explained correctly in Proposition 10.) We get the expression

$$
\left(\begin{array}{l}
n \\
s
\end{array}\right)^{p}=\frac{(-1)^{s p}}{(s !)^{p}} \sum_{t=0}^{1} \sum_{j=s+t}^{s p}(-1)^{j} j ! S_{s, s}(p, j)\left(\begin{array}{c}
n \\
s+t
\end{array}\right)
$$




$$
\begin{aligned}
& +\frac{(-1)^{s p}}{(s !)^{p}} \sum_{t=2}^{3} \sum_{j=s+t}^{s p}(-1)^{j} j !(j-s-t+1) S_{s, s}(p, j)\left(\begin{array}{c}
n+1 \\
s+t
\end{array}\right) \\
& +\frac{(-1)^{s p}}{(s !)^{p}} \sum_{t=4}^{s(p-1)}\left(\sum_{j=s+t}^{s p}(-1)^{j} j !(j-s-t+1) S_{s, s}(p, j)\right)\left(\begin{array}{c}
n+t-2 \\
s+t
\end{array}\right) .
\end{aligned}
$$

And again, we repeat PROCEDURE with (90) as starting point, beginning with the last term and going backwards, up to the fifth term.

After $\left\lfloor\frac{1}{2} s(p-1)\right\rfloor$ times of applying PROCEDURE as described above, we obtain the expression

$$
\begin{aligned}
\left(\begin{array}{c}
n \\
s
\end{array}\right)^{p}= & \frac{(-1)^{s p}}{(s !)^{p}} \sum_{t=0}^{\left\lfloor\frac{1}{2} s(p-1)\right\rfloor} \sum_{j=s+2 t}^{s p}(-1)^{j} j !\left(\begin{array}{c}
j-s-t \\
t
\end{array}\right) S_{s, s}(p, j)\left(\begin{array}{c}
n+t \\
s+2 t
\end{array}\right) \\
& +\frac{(-1)^{s p}}{(s !)^{p}} \sum_{t=0}^{\left\lfloor\frac{1}{2} s(p-1)\right\rfloor} \sum_{j=s+2 t+1}^{s p}(-1)^{j} j !\left(\begin{array}{c}
j-s-t-1 \\
t
\end{array}\right) S_{s, s}(p, j)\left(\begin{array}{c}
n+t \\
s+2 t+1
\end{array}\right) .
\end{aligned}
$$

Let us see a concrete example. For $s=4$ and $p=3$, expression (85) says that

$$
\begin{aligned}
\left(\begin{array}{l}
n \\
4
\end{array}\right)^{3} & =\left(\begin{array}{l}
n \\
4
\end{array}\right)-120\left(\begin{array}{c}
n+1 \\
5
\end{array}\right)+2640\left(\begin{array}{c}
n+2 \\
6
\end{array}\right)-21840\left(\begin{array}{c}
n+3 \\
7
\end{array}\right)+87570\left(\begin{array}{c}
n+4 \\
8
\end{array}\right) \\
& -189000\left(\begin{array}{c}
n+5 \\
9
\end{array}\right)+224700\left(\begin{array}{c}
n+6 \\
10
\end{array}\right)-138600\left(\begin{array}{c}
n+7 \\
11
\end{array}\right)+34650\left(\begin{array}{c}
n+8 \\
12
\end{array}\right) .(92
\end{aligned}
$$

We begin Procedure writing the term $34650\left(\begin{array}{c}n+8 \\ 12\end{array}\right)$ as $34650\left(\left(\begin{array}{c}n+7 \\ 11\end{array}\right)+\left(\begin{array}{c}n+7 \\ 12\end{array}\right)\right)$ and simplifying to

$$
\begin{aligned}
\left(\begin{array}{l}
n \\
4
\end{array}\right)^{3} & =\left(\begin{array}{l}
n \\
4
\end{array}\right)-120\left(\begin{array}{c}
n+1 \\
5
\end{array}\right)+2640\left(\begin{array}{c}
n+2 \\
6
\end{array}\right)-21840\left(\begin{array}{c}
n+3 \\
7
\end{array}\right)+87570\left(\begin{array}{c}
n+4 \\
8
\end{array}\right) \\
& -189000\left(\begin{array}{c}
n+5 \\
9
\end{array}\right)+224700\left(\begin{array}{c}
n+6 \\
10
\end{array}\right)-103950\left(\begin{array}{c}
n+7 \\
11
\end{array}\right)+34650\left(\begin{array}{c}
n+7 \\
12
\end{array}\right) .
\end{aligned}
$$

Now we write the term $103950\left(\begin{array}{c}n+7 \\ 11\end{array}\right)$ of $(93)$ as $103950\left(\left(\begin{array}{c}n+6 \\ 10\end{array}\right)+\left(\begin{array}{c}n+6 \\ 11\end{array}\right)\right)$ and simplify to

$$
\begin{aligned}
\left(\begin{array}{l}
n \\
4
\end{array}\right)^{3}= & \left(\begin{array}{l}
n \\
4
\end{array}\right)-120\left(\begin{array}{c}
n+1 \\
5
\end{array}\right)+2640\left(\begin{array}{c}
n+2 \\
6
\end{array}\right)-21840\left(\begin{array}{c}
n+3 \\
7
\end{array}\right)+87570\left(\begin{array}{c}
n+4 \\
8
\end{array}\right) \\
& -189000\left(\begin{array}{c}
n+5 \\
9
\end{array}\right)+120750\left(\begin{array}{c}
n+6 \\
10
\end{array}\right)-103950\left(\begin{array}{c}
n+6 \\
11
\end{array}\right)+34650\left(\begin{array}{c}
n+7 \\
12
\end{array}\right) .
\end{aligned}
$$

Continuing in this way we obtain

$$
\left(\begin{array}{l}
n \\
4
\end{array}\right)^{3}=\left(\begin{array}{l}
n \\
4
\end{array}\right)+120\left(\begin{array}{c}
n+1 \\
6
\end{array}\right)-2520\left(\begin{array}{c}
n+2 \\
7
\end{array}\right)+19320\left(\begin{array}{c}
n+3 \\
8
\end{array}\right)-68250\left(\begin{array}{c}
n+4 \\
9
\end{array}\right)
$$




$$
+120750\left(\begin{array}{c}
n+5 \\
10
\end{array}\right)-103950\left(\begin{array}{c}
n+6 \\
11
\end{array}\right)+34650\left(\begin{array}{c}
n+7 \\
12
\end{array}\right) .
$$

(This is (89). Observe that the coefficient of the term $\left(\begin{array}{c}n+1 \\ 5\end{array}\right)$ is 0. .) Now we write the last term of the right-hand side of $(94)$ as $34650\left(\left(\begin{array}{c}n+6 \\ 11\end{array}\right)+\left(\begin{array}{c}n+6 \\ 12\end{array}\right)\right)$ and simplify. Then, we repeat this step with the term $(-103950+34650)\left(\begin{array}{c}n+6 \\ 11\end{array}\right)$, and with all the subsequent terms obtained. We get

$$
\begin{aligned}
\left(\begin{array}{l}
n \\
4
\end{array}\right)^{3}= & \left(\begin{array}{l}
n \\
4
\end{array}\right)+120\left(\begin{array}{c}
n+1 \\
6
\end{array}\right)+2520\left(\begin{array}{c}
n+2 \\
8
\end{array}\right)-16800\left(\begin{array}{c}
n+3 \\
9
\end{array}\right) \\
& +51450\left(\begin{array}{c}
n+4 \\
10
\end{array}\right)-69300\left(\begin{array}{c}
n+5 \\
11
\end{array}\right)+34650\left(\begin{array}{c}
n+6 \\
12
\end{array}\right) .
\end{aligned}
$$

(This is (90). Observe that the coefficient of the term $\left(\begin{array}{c}n+2 \\ 7\end{array}\right)$ is 0.) And again, we write the last term of the right-hand side of (95) as $34650\left(\left(\begin{array}{c}n+5 \\ 11\end{array}\right)+\left(\begin{array}{c}n+6 \\ 12\end{array}\right)\right)$ and simplify. And repeat with the subsequent terms to obtain

$$
\begin{aligned}
\left(\begin{array}{l}
n \\
4
\end{array}\right)^{3}= & \left(\begin{array}{l}
n \\
4
\end{array}\right)+120\left(\begin{array}{c}
n+1 \\
6
\end{array}\right)+2520\left(\begin{array}{c}
n+2 \\
8
\end{array}\right)+16800\left(\begin{array}{c}
n+3 \\
10
\end{array}\right) \\
& -34650\left(\begin{array}{c}
n+4 \\
11
\end{array}\right)+34650\left(\begin{array}{c}
n+5 \\
12
\end{array}\right) .
\end{aligned}
$$

One final procedure beginning with the last term of the right-hand side of (96) take us to the formula

$$
\left(\begin{array}{l}
n \\
4
\end{array}\right)^{3}=\left(\begin{array}{l}
n \\
4
\end{array}\right)+120\left(\begin{array}{c}
n+1 \\
6
\end{array}\right)+2520\left(\begin{array}{c}
n+2 \\
8
\end{array}\right)+16800\left(\begin{array}{c}
n+3 \\
10
\end{array}\right)+34650\left(\begin{array}{c}
n+4 \\
12
\end{array}\right) .
$$

The fact that several coefficients are equal to 0 makes (97) a simpler expression than the original one (92). In general we have the following result.

Proposition 10. (a) If $s$ is even or $s$ and $p$ are odd, the following formula holds for any non-negative integer $t$

$$
\sum_{j=s+2 t+1}^{s p}(-1)^{j} j !\left(\begin{array}{c}
j-s-t-1 \\
t
\end{array}\right) S_{s, s}(p, j)=0 .
$$

(b) If $s$ is odd and $p$ is even, the following formula holds for any non-negative integer $t$

$$
\sum_{j=s+2 t+1}^{s p}(-1)^{j} j !\left(\begin{array}{c}
j-s-t-1 \\
t
\end{array}\right) S_{s, s}(p, j)=2 \sum_{j=s+2 t}^{s p}(-1)^{j} j !\left(\begin{array}{c}
j-s-t \\
t
\end{array}\right) S_{s, s}(p, j) .
$$


Proof. (a) Observe that (98) is equivalent to

$$
\begin{aligned}
\sum_{j=s}^{s p}(-1)^{j} j ! S_{s, s}(p, j) & \prod_{l=0}^{t-1}(j-s-t-1-l) \\
& =\sum_{j=s}^{s+t}(-1)^{j} j ! S_{s, s}(p, j) \prod_{l=0}^{t-1}(j-s-t-1-l) .
\end{aligned}
$$

By using Gould's identity (3.50) in [13], we see that

$$
\left(\begin{array}{c}
j-s-t-1 \\
t
\end{array}\right)=\sum_{k=0}^{t}(-1)^{k+t}\left(\begin{array}{c}
2 t-k \\
t
\end{array}\right)\left(\begin{array}{c}
j-s \\
k
\end{array}\right)
$$

which can be written as

$$
\prod_{l=0}^{t-1}(j-s-t-1-l)=\sum_{k=0}^{t} \frac{(-1)^{k}(t+k) !}{k !(t-k) !} \prod_{l=0}^{t-1-k}(j-s-l)
$$

Beginning with the left-hand side of (100) and using (102) we get

$$
\begin{aligned}
& \sum_{j=s}^{s p}(-1)^{j} j ! S_{s, s}(p, j) \prod_{l=0}^{t-1}(j-s-t-1-l) \\
= & \sum_{k=0}^{t} \frac{(-1)^{k}(t+k) !}{k !(t-k) !} \sum_{j=s+t-k}^{s p}(-1)^{j} j ! S_{s, s}(p, j) \prod_{l=0}^{t-1-k}(j-s-l) .
\end{aligned}
$$

By setting $j=s+t-k$ in (83) we see that

$$
S_{s, s}(p, s+t-k)=\frac{(-1)^{s p}}{(t-k) !(s+t-k) !} \sum_{j=s+t-k}^{s p}(-1)^{j} j ! S_{s, s}(p, j) \prod_{l=0}^{t-1-k}(j-s-l) .
$$

According to (104) we can write (103) as

$$
\begin{aligned}
\sum_{j=s}^{s p}(-1)^{j} j ! S_{s, s}(p, j) & \prod_{l=0}^{t-1}(j-s-t-1-l) \\
& =(-1)^{s p} \sum_{k=0}^{t} \frac{(-1)^{k}(t+k) !}{k !}(s+t-k) ! S_{s, s}(p, s+t-k)
\end{aligned}
$$

or

$$
\sum_{j=s}^{s p}(-1)^{j} j ! S_{s, s}(p, j) \prod_{l=0}^{t-1}(j-s-t-1-l)
$$




$$
=(-1)^{s(p+1)+t} \sum_{j=s}^{s+t} \frac{(-1)^{j}(2 t+s-j) !}{(t+s-j) !} j ! S_{s, s}(p, j)
$$

The fact that

$$
\prod_{l=0}^{t-1}(j-s-t-1-l)=(-1)^{t} \frac{(2 t+s-j) !}{(t+s-j) !}
$$

(easy proof, left to the reader), together with the hypothesis that $s$ is even or $s$ and $p$ are odd, give us from (105) the end of the proof of (100).

(b) The case $t=0$ of (99) can be written as

$$
\sum_{j=s}^{s p}(-1)^{j} j ! S_{s, s}(p, j)=s ! S_{s, s}(p, s) .
$$

This is a direct consequence of (58) (with $x=0$ ) and the fact that $p$ is even. So let us consider the cases when $t \geqslant 1$. We can write (99) as

$$
\sum_{j=s+2 t}^{s p}(-1)^{j} j !\left(\left(\begin{array}{c}
j-s-t-1 \\
t
\end{array}\right)+2\left(\begin{array}{c}
j-s-t-1 \\
t-1
\end{array}\right)\right) S_{s, s}(p, j)=0
$$

or

$$
\sum_{j=s+2 t}^{s p}(-1)^{j} j ! S_{s, s}(p, j)(j-s) \prod_{l=0}^{t-2}(j-s-t-1-l)=0 .
$$

To prove (106) is equivalent to prove that

$$
\begin{aligned}
& \sum_{j=s+1}^{s p}(-1)^{j} j ! S_{s, s}(p, j)(j-s) \prod_{l=0}^{t-2}(j-s-t-1-l) \\
= & \sum_{j=s+1}^{s+t}(-1)^{j} j ! S_{s, s}(p, j)(j-s) \prod_{l=0}^{t-2}(j-s-t-1-l) .
\end{aligned}
$$

From Gould's identity (3.50) in [13] we see that

$$
\left(\begin{array}{c}
j-s-t-1 \\
t-1
\end{array}\right)=\sum_{k=0}^{t-1}(-1)^{k+t+1}\left(\begin{array}{c}
2 t-2-k \\
t-1
\end{array}\right)\left(\begin{array}{c}
j-s-1 \\
k
\end{array}\right),
$$

which can be written as

$$
\prod_{l=0}^{t-2}(j-s-t-1-l)=\sum_{k=0}^{t-1} \frac{(-1)^{k}(t-1+k) !}{k !(t-1-k) !} \prod_{l=0}^{t-2-k}(j-s-1-l) .
$$

Beginning with the left-hand side of (107) we can write (by using (108))

$$
\sum_{j=s+1}^{s p}(-1)^{j} j ! S_{s, s}(p, j)(j-s) \prod_{l=0}^{t-2}(j-s-t-1-l)
$$




$$
\begin{aligned}
& =\sum_{j=s+1}^{s p}(-1)^{j} j ! S_{s, s}(p, j)(j-s) \sum_{k=0}^{t-1} \frac{(-1)^{k}(t-1+k) !}{k !(t-1-k) !} \prod_{l=0}^{t-2-k}(j-s-1-l) \\
& =\sum_{k=0}^{t-1} \frac{(-1)^{k}(t-1+k) !}{k !(t-1-k) !} \sum_{j=s+t-k}^{s p}(-1)^{j} j ! S_{s, s}(p, j) \prod_{l=0}^{t-1-k}(j-s-l) .
\end{aligned}
$$

Now we use (104) to write (109) as

$$
\begin{aligned}
& \sum_{j=s+1}^{s p}(-1)^{j} j ! S_{s, s}(p, j)(j-s) \prod_{l=0}^{t-2}(j-s-t-1-l) \\
= & \sum_{k=0}^{t-1} \frac{(-1)^{k+s p}(t-1+k) !}{k !}(t-k)(s+t-k) ! S_{s, s}(p, s+t-k) \\
= & (-1)^{t+s(p-1)} \sum_{j=s+1}^{s+t} \frac{(-1)^{j}(2 t-1-j+s) !}{(t-j+s) !} j !(j-s) S_{s, s}(p, j) .
\end{aligned}
$$

Finally, we use that

$$
\prod_{l=0}^{t-2}(j-s-t-1-l)=(-1)^{t+1} \frac{(2 t-1-j+s) !}{(t-j+s) !}
$$

(proof left to the reader), together with the hypotheses on $s$ and $p$, to obtain from (110) the desired conclusion (107).

Corollary 11. (a) If $s$ is even, or $s$ and $p$ are odd, expression (91) can be written as

$$
\left(\begin{array}{l}
n \\
s
\end{array}\right)^{p}=\frac{(-1)^{s p}}{(s !)^{p}} \sum_{t=0}^{\frac{1}{2} s(p-1)} \sum_{j=s+2 t}^{s p}(-1)^{j} j !\left(\begin{array}{c}
j-s-t \\
t
\end{array}\right) S_{s, s}(p, j)\left(\begin{array}{c}
n+t \\
s+2 t
\end{array}\right) .
$$

(b) If $s$ is odd and $p$ is even, expression (91) can be written as

$$
\begin{aligned}
& \left(\begin{array}{c}
n \\
s
\end{array}\right)^{p} \\
& =\frac{1}{(s !)^{p}} \sum_{t=0}^{\left\lfloor\frac{1}{2} s(p-1)\right\rfloor} \sum_{j=s+2 t}^{s p}(-1)^{j} j !\left(\begin{array}{c}
j-s-t \\
t
\end{array}\right) S_{s, s}(p, j)\left(\left(\begin{array}{c}
n+t \\
s+2 t+1
\end{array}\right)+\left(\begin{array}{c}
n+t+1 \\
s+2 t+1
\end{array}\right)\right) .
\end{aligned}
$$

Proof. Follows from (91) and Proposition 10.

Of course, formulas (111) and (112) have the following versions for partial sums of $\left(\begin{array}{l}n \\ s\end{array}\right)^{p}$ (obtained by taking the convolution $*^{l} 1$ in both sides of (111) and (112), and using (26)) 
(a) If $s$ is even, or $s$ and $p$ are odd, then for any integer $l \geqslant 0$ we have

$$
\left(\begin{array}{c}
n \\
s
\end{array}\right)^{p} *^{l} 1=\frac{(-1)^{s p}}{(s !)^{p}} \sum_{t=0}^{\frac{1}{2} s(p-1)} \sum_{j=s+2 t}^{s p}(-1)^{j} j !\left(\begin{array}{c}
j-s-t \\
t
\end{array}\right) S_{s, s}(p, j)\left(\begin{array}{c}
n+t+l \\
s+2 t+l
\end{array}\right) .
$$

(b) If $s$ is odd and $p$ is even, then for any integer $l \geqslant 0$ we have

$$
\begin{aligned}
& \left(\begin{array}{c}
n \\
s
\end{array}\right)^{p} *^{l} 1 \\
& =\frac{1}{(s !)^{p}} \sum_{t=0}^{\left\lfloor\frac{1}{2} s(p-1)\right\rfloor} \sum_{j=s+2 t}^{s p}(-1)^{j} j !\left(\begin{array}{c}
j-s-t \\
t
\end{array}\right) S_{s, s}(p, j)\left(\left(\begin{array}{c}
n+t+l \\
s+2 t+1+l
\end{array}\right)+\left(\begin{array}{c}
n+t+1+l \\
s+2 t+1+l
\end{array}\right)\right) .
\end{aligned}
$$

Some examples from (113) and (114) are the following (compare them with similar expressions shown in Table 1):

$$
\begin{aligned}
\sum_{k=1}^{n} \sum_{j=1}^{k} \sum_{i=1}^{j}\left(\begin{array}{l}
i \\
2
\end{array}\right)^{2} & =\left(\begin{array}{c}
n+3 \\
5
\end{array}\right)+6\left(\begin{array}{c}
n+4 \\
7
\end{array}\right) . \\
\sum_{i=1}^{n}\left(\begin{array}{l}
i \\
2
\end{array}\right)^{3} & =\left(\begin{array}{c}
n+1 \\
3
\end{array}\right)+24\left(\begin{array}{c}
n+2 \\
5
\end{array}\right)+90\left(\begin{array}{c}
n+3 \\
7
\end{array}\right) . \\
\sum_{j=1}^{n} \sum_{i=1}^{j}\left(\begin{array}{l}
i \\
3
\end{array}\right)^{3} & =\left(\begin{array}{c}
n+2 \\
5
\end{array}\right)+60\left(\begin{array}{c}
n+3 \\
7
\end{array}\right)+630\left(\begin{array}{c}
n+4 \\
9
\end{array}\right)+1680\left(\begin{array}{c}
n+5 \\
11
\end{array}\right) . \\
\sum_{i=1}^{n}\left(\begin{array}{l}
i \\
3
\end{array}\right)^{2} & =\left(\begin{array}{c}
n+1 \\
5
\end{array}\right)+\left(\begin{array}{c}
n+2 \\
5
\end{array}\right)+10\left(\left(\begin{array}{c}
n+2 \\
7
\end{array}\right)+\left(\begin{array}{c}
n+3 \\
7
\end{array}\right)\right) . \\
\sum_{i=1}^{n}\left(\begin{array}{l}
i \\
4
\end{array}\right)^{2} & =\left(\begin{array}{c}
n+1 \\
5
\end{array}\right)+20\left(\begin{array}{c}
n+2 \\
7
\end{array}\right)+70\left(\begin{array}{c}
n+3 \\
9
\end{array}\right) .
\end{aligned}
$$

\section{Acknowledgments}

I thank the referee for calling my attention to some other important works on generalizations of Stirling numbers.

\section{References}

[1] H. Arodź. Comment on 'Combinatorics of boson normal ordering and some applications'. Concepts of Physics, Vol. I: 279-281, 2004. http://merlin.fic.uni.lodz. pl/concepts/2004_3_4/2004_3_4_279.pdf

[2] P. Blasiak. Combinatorics of boson normal ordering and some applications. PhD Thesis: University of Paris VI and Polish Academy of Sciences, Krakow, Poland. arXiv:quant-ph/0507206v2 
[3] P. Blasiak, A. Horzela, K. A. Penson, A. I. Solomon, and G. H. E. Duchamp. Combinatorics and boson normal ordering: A gentle introduction. Am. J. Phys. 75:639-646, 2007.

[4] P. Blasiak, K. A. Penson, and A. I. Solomon. The Boson Normal Ordering Problem and Generalized Bell Numbers. Ann. Comb. 7:127-139, 2003.

[5] N. J. Calkin. Factors of sums of powers of binomial coefficients. Acta Arith 86:17-26, 1998.

[6] L. Carlitz. Weighted Stirling numbers of the first and second kind - I. Fibonacci Quart. 18:147-162, 1980.

[7] Ch. A. Charalambides, Non-central generalized $q$-factorial coefficients and $q$-Stirling numbers, Discrete Math., 275:67-85, 2004.

[8] Ch. A. Charalambides, Enumerative Combinatorics, Chapman \& Hall/CRC, Boca Raton, FL, 2002.

[9] Ch. A. Charalambides and M. Koutras, On the differences of the generalized factorials at an arbitrary point and their combinatorial applications, Discrete Math., 47:183201, 1983.

[10] P. Codara, O. M. D'Antona, and P. Hell. A simple combinatorial interpretation of certain generalized Bell and Stirling numbers. arXiv:1308.1700v1

[11] M. Dziemiańczuk. Generalizing Delannoy numbers via counting weighted lattice paths. Integers 13, \#A54, 2013.

[12] N. Gauthier. Convolving the $m$-th Powers of the Consecutive Integers with the General Fibonacci Sequence Using Carlitz's Weighted Stirling Polynomials of the Second Kind. Fibonacci Quart. 42:306-313, 2004.

[13] H. W. Gould. Combinatorial Identities. Morgantown, W. Va. 1972.

[14] H. W. Gould. Combinatorial Identities: Table I: Intermediate Techniques for Summing Finite Series. From the seven unpublished manuscripts of H. W. Gould. Edited and Compiled by Jocelyn Quaintance, 2010. Available at http://www.math.wvu . edu/ gould/Vol.4.PDF

[15] R. L. Graham, D. E. Knuth, and O. Patashnik, Concrete Mathematics: A Foundation for Computer Science. Second Edition, Addison Wesley, 1993.

[16] Tian-Xiao He, Generalized Stirling Numbers and Generalized Stirling Functions. arXiv:1106.5251v1

[17] Ch. Jordan, Calculus of Finite Differences, Chelsea, New York, 1965.

[18] T. Komatsu, F. Luca, and C. Pita. Generalized poly-Cauchy polynomials and their interpolating functions. Submitted.

[19] M. Koutras, Non-central Stirling numbers and some applications, Discrete Math., 42:73-89, 1982.

[20] W. Lang. Combinatorial Interpretation of Generalized Stirling Numbers. J. Integer Seq. 12, Article 09.3.3, 2009. 
[21] W. Lang. On Generalizations of the Stirling Number Triangles. J. Integer Seq. 3, Article 00.2.4, 2000.

[22] T. Mansour, M. Schork, and M. Shattuck. On a new family of generalized Stirling and Bell numbers. Electron. J. Combin. 18, \#P77, 2011.

[23] M. A. Méndez, P. Blasiak, and K. A. Penson. Combinatorial approach to generalized Bell and Stirling numbers and boson normal ordering problem. J. Math. Phys. 46, Article 083511, 2005.

[24] C. Pita. Some Number Arrays Related to Pascal and Lucas Triangles. J. Integer Seq. 16, Article 13.5.7, 2013.

[25] N. J. A. Sloane. The On-line Encyclopedia of Integer Sequences. http://oeis.org

[26] A. I. Solomon, P. Blasiak, G. Duchamp, A. Horzela, and K. A. Penson. Combinatorial Physics, Normal Order and Model Feynman Graphs. Symmetries in Science XI, pages 527-536. Kluwer Academic Publishers, 2004.

[27] A. I. Solomon, G. Duchamp, P. Blasiak, A. Horzela, and K. A. Penson. Normal Order: Combinatorial Graphs. arXiv:quant-ph/0402082v1 